\title{
Finite Rank Perturbations, Scattering Matrices and Inverse Problems
}

\author{
Jussi Behrndt* \\ Technische Universität Berlin \\ Institut für Mathematik \\ MA 6-4, Straße des 17. Juni 136 \\ D-10623 Berlin, Germany \\ Mark M. Malamud ${ }^{\dagger}$ \\ Donetsk National University \\ Department of Mathematics \\ Universitetskaya 24 \\ 83055 Donetsk, Ukraine \\ Hagen Neidhardt ${ }^{\ddagger}$ \\ Weierstraß-Institut für \\ Angewandte Analysis und Stochastik \\ Mohrenstr. 39 \\ D-10117 Berlin, Germany
}

February 19, 2009

Dedicated to the memory of our friend Peter Jonas (18.7.1941 - 18.7.2007)

\begin{abstract}
In this paper the scattering matrix of a scattering system consisting of two selfadjoint operators with finite dimensional resolvent difference is expressed in terms of a matrix Nevanlinna function. The problem is embedded into an extension theoretic framework and the theory of boundary triplets and associated Weyl functions for (in general nondensely defined) symmetric operators is applied. The representation results are extended to dissipative scattering systems and an explicit solution of an inverse scattering problem for the Lax-Phillips scattering matrix is presented.
\end{abstract}

Subject classification: Primary 47A40; Secondary 81U40, 47A55, 47B44

Keywords: Scattering system, scattering matrix, boundary triplet, Weyl function, dissipative operator, Lax-Phillips scattering

\footnotetext{
*E-mail: behrndt@math.tu-berlin.de

${ }^{\dagger}$ E-mail: mmm@telenet.dn.ua

‡E-mail: neidhardt@wias-berlin.de
} 


\section{Introduction}

Let $A$ and $B$ be selfadjoint operators in a Hilbert space $\mathfrak{H}$ and assume that the difference of the resolvents

$$
(B-\lambda)^{-1}-(A-\lambda)^{-1}, \quad \lambda \in \rho(A) \cap \rho(B),
$$

is a trace class operator. Then it is well known that the wave operators $W_{ \pm}(B, A)$ exist; they are isometries mapping the absolutely continuous subspace of $A$ onto that of $B$. The scattering operator $S_{A B}:=W_{+}(B, A)^{*} W_{-}(B, A)$ of the scattering system $\{A, B\}$ commutes with $A$ and is unitary on the absolutely continuous subspace of $A$. Therefore $S_{A B}$ is unitarily equivalent to a multiplication operator induced by a family of unitary operators $\left\{S_{A B}(\lambda)\right\}_{\lambda \in \mathbb{R}}$ in the spectral representation of the absolutely continuous part of $A$. The family $\left\{S_{A B}(\lambda)\right\}_{\lambda \in \mathbb{R}}$ is called the scattering matrix of $\{A, B\}$.

One of the main objectives of this paper is to represent the scattering matrix of the scattering system $\{A, B\}$ with the help of an associated Nevanlinna function $M(\cdot)$. We restrict ourselves to the special case of finite rank perturbations in resolvent sense, i.e., it is assumed that the difference of the resolvents in (1.1) is a rank $n$ operator, where $n<\infty$. In this case the Nevanlinna function $M(\cdot)$ will be an $n \times n$-matrix function and it will be shown in Theorem 3.1 that the scattering matrix $\left\{S_{A B}(\lambda)\right\}_{\lambda \in \mathbb{R}}$ is given by

$$
S_{A B}(\lambda)=I-2 i \sqrt{\Im m(M(\lambda+i 0))} M(\lambda+i 0)^{-1} \sqrt{\Im m(M(\lambda+i 0))}
$$

for a.e. $\lambda \in \mathbb{R}$. This representation is a generalization of a recent result of the authors from 8 and an earlier different (unitarily equivalent) expression found by V.M. Adamyan and B.S. Pavlov in 5 . The formula (1.2) is obtained by embedding the scattering problem into an extension theoretic framework. More precisely, we consider the (in general nondensely defined) closed symmetric operator $S=A \cap B$ which has finite equal deficiency indices $(n, n)$. The adjoint $S^{*}$ is defined in the sense of linear relations and a so-called boundary triplet $\Pi=\left\{\mathbb{C}^{n}, \Gamma_{0}, \Gamma_{1}\right\}$ for $S^{*}$ is chosen in such a way that the selfadjoint extensions of $S$ corresponding to the boundary mappings $\Gamma_{0}$ and $\Gamma_{1}$ coincide with $A$ and $B$, respectively. The function $M(\cdot)$ in (1.2) is the Weyl function associated to this boundary triplet - an abstract analogon of the classical Titchmarsh-Weyl $m$-function from singular Sturm-Liouville theory - and contains the spectral information of the operator $A$.

Besides selfadjoint scattering systems we also consider so-called maximal dissipative scattering systems $\{A, B\}$, that is, $A$ is still a selfadjoint operator in $\mathfrak{H}$ but $B$ is only assumed to be maximal dissipative, i.e., $\Im m(B f, f) \leq 0$ and the spectrum of $B$ is contained in $\mathbb{C}_{-} \cup \mathbb{R}$. As above we treat only the case of finite rank perturbations in resolvent sense. Following [7, 25, 26, 27] a minimal selfadjoint dilation $L$ of $B$ in the direct sum $\mathfrak{H} \oplus L^{2}\left(\mathbb{R}, \mathbb{C}^{n}\right)$ is constructed and a natural larger selfadjoint scattering system $\{K, L\}$ in $\mathfrak{H} \oplus L^{2}\left(\mathbb{R}, \mathbb{C}^{n}\right)$ is considered. From Theorem 3.1 and Theorem 3.2 we obtain a representation of the scattering matrix $\left\{S_{K L}(\lambda)\right\}_{\lambda \in \mathbb{R}}$ which is closely related to the representations found earlier in [7. We emphasize that the lower right corner of $\left\{S_{K L}(\lambda)\right\}_{\lambda \in \mathbb{R}}$ in Proposition 4.4 can be interpreted as the Lax-Phillips scattering matrix $\left\{S^{L P}(\lambda)\right\}_{\lambda \in \mathbb{R}}$ of the Lax-Phillips scattering system $\left\{L, \mathcal{D}_{-}, \mathcal{D}_{+}\right\}$, where the incoming and outcoming subspaces $\mathcal{D}_{-}$and $\mathcal{D}_{+}$are $L^{2}\left(\mathbb{R}_{-}, \mathbb{C}^{n}\right)$ and $L^{2}\left(\mathbb{R}_{+}, \mathbb{C}^{n}\right)$, respectively. This 
also implies the well known relation $S^{L P}(\lambda)=\Theta_{B}(\lambda-i 0)^{*}$ between the LaxPhillips scattering matrix and the characteristic function $\Theta_{B}(\cdot)$ of the maximal dissipative operator $B$ found by V.M. Adamyan and D.Z. Arov in 1, 2, 3, 4.

As an application of our approach on finite rank perturbations and maximal dissipative scattering systems we prove an inverse result in Section 5 Let $W(\cdot)$ be a purely contractive analytic matrix function on $\mathbb{C}_{+}$. Under some mild additional assumptions it is shown in Theorem 5.1 that the limit $\{W(\lambda+i 0)\}_{\lambda \in \mathbb{R}}$ can be regarded as the Lax-Phillips scattering matrix of a suitable chosen LaxPhillips scattering system. We point out that this statement can be obtained immediately in a more abstract and general form by combining the results of B. Sz.-Nagy and C. Foias in [27, Section VI] with the results of V.M. Adamyan and D.Z. Arov in 1, 2, 3, 4. However, our approach leads to a more explicit solution of the inverse problem, in particular, we find a maximal dissipative multiplication operator $B$ in an $L^{2}$-space and a minimal selfadjoint dilation $L$ of $B$ such that the Lax-Phillips scattering matrix of $\left\{L, \mathcal{D}_{-}, \mathcal{D}_{+}\right\}$coincides with the limit of the given purely contractive analytic matrix function $W(\cdot)$; cf. Corollary 5.2

The paper is organized as follows. In Section 2 we give a brief introduction in the theory of boundary triplets for (in general nondensely defined) closed symmetric operators. In particular, we show how a boundary triplet for the intersection $S=A \cap B$ of two selfadjoint operators $A$ and $B$ with a finite dimensional resolvent difference can be chosen. Section 3 is devoted to the representation of the scattering matrix for a scattering system $\{A, B\}$ with finite rank resolvent difference and in Section 4 the results are extended to the case where the operator $B$ is only maximal dissipative. With the help of these results we propose a solution for the inverse scattering problem in Section 5 . For the convenience of the reader we add an Appendix on direct integrals, spectral representations and scattering matrices.

Notation. The Hilbert spaces in this paper are usually denoted by $\mathfrak{H}, \mathfrak{K}$ and $\mathcal{H}$; they are all assumed to be separable. The symbols $\operatorname{span}\{\cdot\}$ and $\operatorname{clospan}\{\cdot\}$ are used for the linear span and closed linear span, respectively, of a set. The algebra of everywhere defined bounded linear operators on a Hilbert space $\mathfrak{H}$ with values in a Hilbert space $\mathfrak{K}$ is denoted by $[\mathfrak{H}, \mathfrak{K}]$; we write $[\mathfrak{H}]$ if $\mathfrak{K}=\mathfrak{H}$. By $\mathcal{F}_{n}(\mathfrak{H})$ we denote the subset of $[\mathfrak{H}]$ that consists of linear operators with range of dimension $n \in \mathbb{N}$. The absolutely continuous part of a selfadjoint operator $A$ in $\mathfrak{H}$ is denoted by $A^{a c}$, the corresponding subspace by $\mathfrak{H}^{a c}(A)$. The symbols $\rho(\cdot), \sigma(\cdot), \sigma_{p}(\cdot), \sigma_{c}(\cdot), \sigma_{r}(\cdot)$ stand for the resolvent set, the spectrum, the point, continuous and residual spectrum, respectively. By $E(\cdot)$ and $\Sigma(\cdot)$ we denote operator-valued measures defined on the algebra of Borel sets $\mathcal{B}(\mathbb{R})$ of the real axis $\mathbb{R}$. Usually, the symbol $E(\cdot)$ is reserved for orthogonal operator-valued measures. 


\section{Selfadjoint and maximal dissipative exten- sions of nondensely defined symmetric opera- tors}

\subsection{Linear relations}

Let $(\mathfrak{H},(\cdot, \cdot))$ be a separable Hilbert space. A (closed) linear relation $T$ in $\mathfrak{H}$ is a (closed) linear subspace of the Cartesian product space $\mathfrak{H} \times \mathfrak{H}$. The set of closed linear relations in $\mathfrak{H}$ is denoted by $\widetilde{\mathcal{C}}(\mathfrak{H})$. Linear operators in $\mathfrak{H}$ will always be identified with linear relations via their graphs. The elements of a linear relation $T$ are pairs denoted by $\widehat{f}=\left\{f, f^{\prime}\right\} \in T, f, f^{\prime} \in \mathfrak{H}$, and the domain, kernel, range, and the multi-valued part of $T$ are defined as

$$
\begin{aligned}
\operatorname{dom}(T) & =\left\{f \in \mathfrak{H}:\left\{f, f^{\prime}\right\} \in T\right\}, \quad \operatorname{ker}(T)=\{f \in \mathfrak{H}:\{f, 0\} \in T\}, \\
\operatorname{ran}(T) & =\left\{f^{\prime} \in \mathfrak{H}:\left\{f, f^{\prime}\right\} \in T\right\}, \quad \operatorname{mul}(T)=\left\{f^{\prime} \in \mathfrak{H}:\left\{0, f^{\prime}\right\} \in T\right\},
\end{aligned}
$$

respectively. Note that $T$ is an operator if and only if $\operatorname{mul}(T)=\{0\}$. A point $\lambda$ belongs to the resolvent set $\rho(T)$ of a closed linear relation $T$ if $(T-\lambda)^{-1}$ is an everywhere defined bounded operator in $\mathfrak{H}$. The spectrum $\sigma(T)$ of $T$ is the complement of $\rho(T)$ in $\mathbb{C}$.

A linear relation $T$ in $\mathfrak{H}$ is called dissipative if $\operatorname{Im}\left(f^{\prime}, f\right) \leq 0$ holds for all $\left\{f, f^{\prime}\right\} \in T$. A dissipative relation $T$ is said to be maximal dissipative if there exists no proper dissipative extension of $T$ in $\mathfrak{H}$. It can be shown that a dissipative relation $T$ is maximal dissipative if and only if $\mathbb{C}_{+} \subset \rho(T)$ holds.

The adjoint $T^{*}$ of a linear relation $T$ in $\mathfrak{H}$ is a closed linear relation in $\mathfrak{H}$ defined by

$$
T^{*}:=\left\{\left\{g, g^{\prime}\right\}:\left(f^{\prime}, g\right)=\left(f, g^{\prime}\right) \text { for all }\left\{f, f^{\prime}\right\} \in T\right\} \text {. }
$$

Observe that this definition extends the usual definition of the adjoint operator and that $\operatorname{mul}\left(T^{*}\right)=(\operatorname{dom}(T))^{\perp}$ holds. In particular, $T^{*}$ is an operator if and only if $T$ is densely defined. A linear relation $T$ in $\mathfrak{H}$ is called symmetric (selfadjoint) if $T \subset T^{*}\left(T=T^{*}\right.$, respectively). It follows from the polarization identity that $T$ is symmetric if and only if $\left(f^{\prime}, f\right) \in \mathbb{R}$ for all $\left\{f, f^{\prime}\right\} \in T$.

A (possibly nondensely defined) symmetric operator $S$ in $\mathfrak{H}$ is said to be simple if there is no nontrivial subspace in $\mathfrak{H}$ which reduces $S$ to a selfadjoint operator. It is well known that every symmetric operator $S$ can be written as the direct orthogonal sum $\widehat{S} \oplus S_{s}$ of a simple symmetric operator $\widehat{S}$ in the Hilbert space

$$
\widehat{\mathfrak{H}}=\operatorname{clospan}\left\{\operatorname{ker}\left(S^{*}-\lambda\right): \lambda \in \mathbb{C} \backslash \mathbb{R}\right\}
$$

and a selfadjoint operator $S_{s}$ in $\mathfrak{H} \ominus \widehat{\mathfrak{H}}$.

\subsection{Boundary triplets for nondensely defined symmetric operators}

Let in the following $S$ be a (not necessarily densely defined) closed symmetric operator in the separable Hilbert space $\mathfrak{H}$ with equal deficiency indices

$$
n_{ \pm}(S)=\operatorname{dim}\left(\operatorname{ran}(S \pm i)^{\perp}\right)=\operatorname{dim}\left(\operatorname{ker}\left(S^{*} \mp i\right)\right) \leq \infty .
$$


If $\operatorname{dom}(S)$ is not dense in $\mathfrak{H}$ the adjoint $S^{*}$ exists only in the sense of linear relations and is defined as in (2.1). Therefore, if $S$ is not densely defined the closed extensions $S^{\prime} \subset S^{*}$ of $S$ in $\mathfrak{H}$ may have nontrivial multi-valued parts. However, the operator $S$ admits also closed extensions in $\mathfrak{H}$ which are operators. We will use the concept of boundary triplets for the description of the closed extensions $S^{\prime} \subset S^{*}$ of $S$ in $\mathfrak{H}$; see, e.g., [12, 13, 14, 15, 18, 23. This concept also provides a convenient criterion to check whether $S^{\prime}$ is an operator or not; cf. (2.4).

Definition 2.1 A triplet $\Pi=\left\{\mathcal{H}, \Gamma_{0}, \Gamma_{1}\right\}$ is called a boundary triplet for $S^{*}$ if $\mathcal{H}$ is a Hilbert space and $\Gamma_{0}, \Gamma_{1}: S^{*} \rightarrow \mathcal{H}$ are linear mappings such that the abstract Green's identity

$$
\left(f^{\prime}, g\right)-\left(f, g^{\prime}\right)=\left(\Gamma_{1} \hat{f}, \Gamma_{0} \hat{g}\right)-\left(\Gamma_{0} \hat{f}, \Gamma_{1} \hat{g}\right)
$$

holds for all $\widehat{f}=\left\{f, f^{\prime}\right\}, \widehat{g}=\left\{g, g^{\prime}\right\} \in S^{*}$ and the mapping $\Gamma:=\left(\Gamma_{0}, \Gamma_{1}\right)^{\top}$ : $S^{*} \rightarrow \mathcal{H} \oplus \mathcal{H}$ is surjective.

We refer to 14, 15, 18, 23 for a detailed study of boundary triplets and recall only some important facts. First of all a boundary triplet $\Pi=\left\{\mathcal{H}, \Gamma_{0}, \Gamma_{1}\right\}$ for $S^{*}$ exists (but is not unique) since the deficiency indices $n_{ \pm}(S)$ of $S$ are assumed to be equal. Then $n_{ \pm}(S)=\operatorname{dim} \mathcal{H}$ holds. A standard construction of a boundary triplet will be given in the proof of Proposition 2.2.

Let $\Pi=\left\{\mathcal{H}, \Gamma_{0}, \Gamma_{1}\right\}$ be a boundary triplet for $S^{*}$ and let $\operatorname{Ext}(S)$ be the set of all closed extensions $S^{\prime} \subset S^{*}$ of $S$. Then $S=\operatorname{ker}(\Gamma)$ and the mapping

$$
\Theta \mapsto S_{\Theta}:=\Gamma^{-1} \Theta=\left\{\widehat{f} \in S^{*}:\left\{\Gamma_{0} \widehat{f}, \Gamma_{1} \widehat{f}\right\} \in \Theta\right\}
$$

establishes a bijective correspondence between the set $\widetilde{\mathcal{C}}(\mathcal{H})$ of closed linear relations in $\mathfrak{H}$ and the set of closed extensions $S_{\Theta} \in \operatorname{Ext}(S)$ of $S$. We note that the right-hand side of (2.3) can also be written as $\operatorname{ker}\left(\Gamma_{1}-\Theta \Gamma_{0}\right)$ where the sum and product is interpreted in the sense of linear relations. Since $\left(S_{\Theta}\right)^{*}=S_{\Theta^{*}}$ holds for every $\Theta \in \widetilde{\mathcal{C}}(\mathcal{H})$ it follows that $S_{\Theta}$ is symmetric (selfadjoint) if and only if $\Theta$ is symmetric (selfadjoint, respectively). In particular, the extensions $A_{0}:=\operatorname{ker}\left(\Gamma_{0}\right)$ and $A_{1}:=\operatorname{ker}\left(\Gamma_{1}\right)$ are selfadjoint. The selfadjoint operator or relation $A_{0}$ will often play the role of a fixed selfadjoint extension of $S$ in $\mathfrak{H}$. Furthermore, an extension $S_{\Theta} \in \operatorname{Ext}(S)$ is dissipative (maximal dissipative) if and only if $\Theta$ is dissipative (maximal dissipative, respectively). We note that $S_{\Theta}$ in (2.3) is an operator if and only if

$$
\Theta \cap\left\{\left\{\Gamma_{0} \widehat{f}, \Gamma_{1} \widehat{f}\right\}: \widehat{f}=\left\{0, f^{\prime}\right\} \in S^{*}\right\}=\{0\} .
$$

The following proposition is a consequence of the basic properties of boundary triplets and results from [14, 15, 23. Since it plays an important role in this paper we give a complete proof for the convenience of the reader. We also note that the statement remains true if $A$ and $B$ are linear relations instead of operators. Recall that $\mathcal{F}_{n}(\mathfrak{H}), n \in \mathbb{N}$, is the set of finite dimensional operators in $\mathfrak{H}$ with ranges of dimension $n$, i.e.,

$$
\mathcal{F}_{n}(\mathfrak{H})=\{T \in[\mathfrak{H}]: \operatorname{dim}(\operatorname{ran}(T))=n\} .
$$


Proposition 2.2 Let $A$ be a selfadjoint operator and let $B$ be a maximal dissipative operator in $\mathfrak{H}$. Assume that

$$
(B-\lambda)^{-1}-(A-\lambda)^{-1} \in \mathcal{F}_{n}(\mathfrak{H})
$$

holds for some (and hence for all) $\lambda \in \mathbb{C}_{+}$. Then the closed symmetric operator $S:=A \cap B$ has finite deficiency indices $(n, n)$ in $\mathfrak{H}$ and there exists a boundary triplet $\Pi=\left\{\mathbb{C}^{n}, \Gamma_{0}, \Gamma_{1}\right\}$ for $S^{*}$ and a dissipative $n \times n$-matrix $D$ such that $A=\operatorname{ker}\left(\Gamma_{0}\right)$ and $B=\operatorname{ker}\left(\Gamma_{1}-D \Gamma_{0}\right)$ holds.

Proof. Let $\lambda_{0} \in \rho(A) \cap \rho(B)$ and let $n \in \mathbb{N},\left\{e_{1}, \ldots, e_{n}\right\}$ and $\left\{f_{1}, \ldots, f_{n}\right\}$ be linearly independent vectors such that

$$
\left(B-\lambda_{0}\right)^{-1}-\left(A-\lambda_{0}\right)^{-1}=\sum_{i=1}^{n}\left(\cdot, e_{i}\right) f_{i} .
$$

The operator $S=A \cap B$, that is,

$$
S f=A f=B f, \quad \operatorname{dom} S=\{f \in \operatorname{dom} A \cap \operatorname{dom} B: A f=B f\},
$$

is a (in general non-densely defined) symmetric operator in $\mathfrak{H}$ and it is easy to check that

$$
\left(S-\lambda_{0}\right)^{-1}=\left(A-\lambda_{0}\right)^{-1} \cap\left(B-\lambda_{0}\right)^{-1}
$$

holds. The intersection in (2.6) is understood in the sense of linear relations. Hence (2.5) and (2.6) imply $\left.\left.\operatorname{dim}\left(\operatorname{ran}\left(A-\lambda_{0}\right)^{-1}\right) / \operatorname{ran}\left(S-\lambda_{0}\right)^{-1}\right)\right)=n$. Therefore $\operatorname{dim}(A / S)=n$ and $S$ has deficiency indices $(n, n)$. Note that $\left(S-\lambda_{0}\right)^{-1}$ is defined on the subspace $\mathfrak{H} \ominus \operatorname{span}\left\{e_{1}, \ldots, e_{n}\right\}$ which has codimension $n$ in $\mathfrak{H}$.

It is not difficult to verify that $S^{*}$ coincides with the direct sum of the subspaces $A$ and

$$
\widehat{\mathcal{N}}_{\lambda_{0}}=\left\{\left\{f_{\lambda_{0}}, \lambda_{0} f_{\lambda_{0}}\right\}: f_{\lambda_{0}} \in \mathcal{N}_{\lambda_{0}}=\operatorname{ker}\left(S^{*}-\lambda_{0}\right)\right\} .
$$

Let us decompose the elements $\widehat{f} \in S^{*}$ accordingly, i.e.,

$$
\widehat{f}=\left\{f, f^{\prime}\right\}=\left\{f_{A}+f_{\lambda_{0}}, A f_{A}+\lambda_{0} f_{\lambda_{0}}\right\}, \quad f_{A} \in \operatorname{dom} A, f_{\lambda_{0}} \in \mathcal{N}_{\lambda_{0}},
$$

and denote by $P_{0}$ the orthogonal projection onto the closed subspace $\mathcal{N}_{\lambda_{0}}$. Then $\Pi=\left\{\mathcal{N}_{\lambda_{0}}, \Gamma_{0}, \Gamma_{1}\right\}$, where

$$
\Gamma_{0} \widehat{f}:=f_{\lambda_{0}} \quad \text { and } \quad \Gamma_{1} \widehat{f}:=P_{0}\left(\left(A-\bar{\lambda}_{0}\right) f_{A}+\lambda_{0} f_{\lambda_{0}}\right),
$$

$\widehat{f} \in S^{*}$, is a boundary triplet with $A=A_{0}:=\operatorname{ker}\left(\Gamma_{0}\right)$. In fact, for $\widehat{f}$ as in (2.7) and $\widehat{g}=\left\{g, g^{\prime}\right\}=\left\{g_{A}+g_{\lambda_{0}}, A g_{A}+\lambda_{0} g_{\lambda_{0}}\right\}$ we obtain from $\left(A f_{A}, g_{A}\right)=\left(f_{A}, A g_{A}\right)$ that

$$
\begin{aligned}
\left(f^{\prime}, g\right)-\left(f, g^{\prime}\right) & =\left(\left(A-\bar{\lambda}_{0}\right) f_{A}+\lambda_{0} f_{\lambda_{0}}, g_{\lambda_{0}}\right)-\left(f_{\lambda_{0}},\left(A-\bar{\lambda}_{0}\right) g_{A}+\lambda_{0} g_{\lambda_{0}}\right) \\
& =\left(\Gamma_{1} \widehat{f}, \Gamma_{0} \widehat{g}\right)-\left(\Gamma_{0} \widehat{f}, \Gamma_{1} \widehat{g}\right)
\end{aligned}
$$

holds. The surjectivity of the mapping $\Gamma=\left(\Gamma_{0}, \Gamma_{1}\right)^{\top}: S^{*} \rightarrow \mathcal{N}_{\lambda_{0}} \oplus \mathcal{N}_{\lambda_{0}}$ follows from $\bar{\lambda}_{0} \in \rho(A)$ since for $x, x^{\prime} \in \mathcal{N}_{\lambda_{0}}$ we can choose $f_{A} \in \operatorname{dom} A$ such that $\left(A-\bar{\lambda}_{0}\right) f_{A}=x^{\prime}-\lambda_{0} x$ holds. Then obviously $\widehat{f}:=\left\{f_{A}+x, A f_{A}+\lambda_{0} x\right\}$ satisfies 
$\Gamma \widehat{f}=\left(x, x^{\prime}\right)^{\top}$. Moreover, from the definition of $\Gamma_{0}$ we immediately obtain that the extension $A_{0}=\operatorname{ker}\left(\Gamma_{0}\right)$ coincides with the operator $A$. As the deficiency indices of $S$ are $(n, n)$ we can identify $\mathcal{N}_{\lambda_{0}}$ with $\mathbb{C}^{n}$.

Since $B$ is a maximal dissipative extension of the symmetric operator $S$, $B \in \operatorname{Ext}(S)$. Hence $B \subset \operatorname{dom}(\Gamma)$ and the linear relation

$$
D:=\Gamma B=\left\{\left\{\Gamma_{0} \widehat{f}, \Gamma_{1} \widehat{f}\right\}: \widehat{f}=\{f, B f\} \in B\right\}
$$

is maximal dissipative in $\mathbb{C}^{n}$ and $B$ coincides with the maximal dissipative extension $S_{D}$ via (2.3). We claim that $D$ is a matrix, i.e., $\operatorname{mul}(D)=\{0\}$. In fact, assume that $D$ is multi-valued, that is, there exists $\widehat{f}=\{f, B f\} \in B$ such that $\left\{0, \Gamma_{1} \widehat{f}\right\} \in D$ with $\Gamma_{1} \widehat{f} \neq 0$. In particular, $\Gamma_{0} \widehat{f}=0$, i.e., $\widehat{f} \in A_{0}=A$ and therefore $\widehat{f} \in A \cap B=S=\operatorname{ker}\left(\Gamma_{0}, \Gamma_{1}\right)^{\top}$, however, this is a contradiction. Thus $D$ is a dissipative $n \times n$-matrix and it follows from (2.3) that $B=\operatorname{ker}\left(\Gamma_{1}-D \Gamma_{0}\right)$ holds.

\subsection{Weyl functions and Krein's formula}

Again let $S$ be a (in general nondensely defined) closed symmetric operator in $\mathfrak{H}$ with equal deficiency indices as in the previous section. If $\lambda \in \mathbb{C}$ is a point of regular type of $S$, i.e., $(S-\lambda)^{-1}$ is a bounded operator, we denote the defect subspace of $S$ at $\lambda$ by $\mathcal{N}_{\lambda}=\operatorname{ker}\left(S^{*}-\lambda\right)$ and we agree to write

$$
\widehat{\mathcal{N}}_{\lambda}=\left\{\{f, \lambda f\}: f \in \mathcal{N}_{\lambda}\right\} \subset S^{*} .
$$

Let $\Pi=\left\{\mathcal{H}, \Gamma_{0}, \Gamma_{1}\right\}$ be a boundary triplet for $S^{*}$ and let $A_{0}=\operatorname{ker}\left(\Gamma_{0}\right)$ be the fixed selfadjoint extension of $S$. Recall that for every $\lambda \in \rho\left(A_{0}\right)$ the relation $S^{*}$ is the direct sum of the selfadjoint relation $A_{0}$ and $\widehat{\mathcal{N}}_{\lambda}$ and denote by $\pi_{1}$ the orthogonal projection onto the first component of $\mathfrak{H} \oplus \mathfrak{H}$. The operator valued functions

$$
\gamma(\cdot): \rho\left(A_{0}\right) \rightarrow[\mathcal{H}, \mathfrak{H}], \quad \lambda \mapsto \gamma(\lambda)=\pi_{1}\left(\Gamma_{0} \uparrow \widehat{\mathcal{N}}_{\lambda}\right)^{-1}
$$

and

$$
M(\cdot): \rho\left(A_{0}\right) \rightarrow[\mathcal{H}], \quad \lambda \mapsto M(\lambda)=\Gamma_{1}\left(\Gamma_{0}\left\lceil\widehat{\mathcal{N}}_{\lambda}\right)^{-1}\right.
$$

are called the $\gamma$-field and the Weyl function, respectively, corresponding to the boundary triplet $\Pi=\left\{\mathcal{H}, \Gamma_{0}, \Gamma_{1}\right\}$; see, e.g., $13,14,15,23$. It can be shown that both $\gamma(\cdot)$ and $M(\cdot)$ are holomorphic on $\rho\left(A_{0}\right)$ and that the identities

$$
\gamma(\mu)=\left(I+(\mu-\lambda)\left(A_{0}-\mu\right)^{-1}\right) \gamma(\lambda), \quad \lambda, \mu \in \rho\left(A_{0}\right),
$$

and

$$
M(\lambda)-M(\mu)^{*}=(\lambda-\bar{\mu}) \gamma(\mu)^{*} \gamma(\lambda), \quad \lambda, \mu \in \rho\left(A_{0}\right),
$$

are valid; see [14, 23]. The identity (2.9) yields that $M(\cdot)$ is a $[\mathcal{H}]$-valued Nevanlinna function, that is, $M(\cdot)$ is holomorphic on $\mathbb{C} \backslash \mathbb{R}, \Im \mathrm{m}(M(\lambda))$ is a nonnegative operator for all $\lambda \in \mathbb{C}_{+}$and $M(\lambda)=M(\bar{\lambda})^{*}$ holds for all $\lambda \in \mathbb{C} \backslash \mathbb{R}$. Moreover, it follows from (2.9) that $0 \in \rho(\Im \mathrm{m}(M(\lambda)))$ for all $\lambda \in \mathbb{C} \backslash \mathbb{R}$ and, in particular,

$$
\frac{\Im \mathrm{m}(M(\lambda))}{\Im \mathrm{m}(\lambda)}=\gamma(\lambda)^{*} \gamma(\lambda), \quad \lambda \in \mathbb{C} \backslash \mathbb{R} .
$$

The following inverse result is essentially a consequence of [21], see also [15, 23]. 
Theorem 2.3 Let $M: \mathbb{C} \backslash \mathbb{R} \rightarrow[\mathcal{H}]$ be a Nevanlinna function such that $0 \in \rho(\Im \mathrm{m}(M(\lambda)))$ for some (and hence for all) $\lambda \in \mathbb{C} \backslash \mathbb{R}$ and assume that the condition

$$
\lim _{\eta \rightarrow+\infty} \frac{1}{\eta}(M(i \eta) h, h)=0
$$

holds for all $h \in \mathcal{H}$. Then there exists a separable Hilbert space $\mathfrak{H}$, a closed simple symmetric operator $S$ in $\mathfrak{H}$ and a boundary triplet $\Pi=\left\{\mathcal{H}, \Gamma_{0}, \Gamma_{1}\right\}$ for the adjoint relation $S^{*}$ such that $A_{0}=\operatorname{ker}\left(\Gamma_{0}\right)$ is a selfadjoint operator and the Weyl function of $\Pi$ coincides with $M(\cdot)$ on $\mathbb{C} \backslash \mathbb{R}$. The symmetric operator $S$ is densely defined if and only if the conditions (2.11) and

$$
\lim _{\eta \rightarrow+\infty} \eta \Im \mathrm{m}(M(i \eta) h, h)=\infty, \quad h \in \mathcal{H}, h \neq 0,
$$

are satisfied.

The spectrum and the resolvent set of closed extensions in $\operatorname{Ext}(S)$ can be described with the help of the Weyl function. More precisely, if $S_{\Theta} \in \operatorname{Ext}(S)$ is the extension corresponding to $\Theta \in \widetilde{\mathcal{C}}(\mathcal{H})$ via (2.3), then a point $\lambda \in \rho\left(A_{0}\right)$ belongs to $\rho\left(S_{\Theta}\right)\left(\sigma_{i}\left(S_{\Theta}\right), i=p, c, r\right)$ if and only if $0 \in \rho(\Theta-M(\lambda))(0 \in$ $\sigma_{i}(\Theta-M(\lambda)), i=p, c, r$, respectively). Moreover, for $\lambda \in \rho\left(A_{0}\right) \cap \rho\left(S_{\Theta}\right)$ the well-known resolvent formula

$$
\left(S_{\Theta}-\lambda\right)^{-1}=\left(A_{0}-\lambda\right)^{-1}+\gamma(\lambda)(\Theta-M(\lambda))^{-1} \gamma(\bar{\lambda})^{*}
$$

holds, see 14, 23. Formula (2.12) and Proposition 2.2 imply the following statement which will be used in Section 4

Corollary 2.4 Let $A$ be a selfadjoint operator and let $B$ be a maximal dissipative operator in $\mathfrak{H}$ such that

$$
(B-\lambda)^{-1}-(A-\lambda)^{-1} \in \mathcal{F}_{n}(\mathfrak{H})
$$

holds for some (and hence for all) $\lambda \in \mathbb{C}_{+}$. Let $\Pi=\left\{\mathbb{C}^{n}, \Gamma_{0}, \Gamma_{1}\right\}$ be the boundary triplet from Proposition 2.2 such that $A=\operatorname{ker}\left(\Gamma_{0}\right)$ and $B=\operatorname{ker}\left(\Gamma_{1}-D \Gamma_{0}\right)$ holds with some dissipative $n \times n$-matrix $D$ and denote the $\gamma$-field and the Weyl function of $\Pi$ by $\gamma(\cdot)$ and $M(\cdot)$, respectively. Then

$$
(B-\lambda)^{-1}-(A-\lambda)^{-1}=\gamma(\lambda)(D-M(\lambda))^{-1} \gamma(\bar{\lambda})^{*}
$$

holds for all $\lambda \in \rho(B) \cap \rho(A)$.

If the maximal dissipative operator $B$ in Proposition 2.2 and Corollary 2.4 is even selfadjoint the representation of the resolvent difference in (2.13) can be further simplified.

Corollary 2.5 Let $A$ and $B$ be selfadjoint operators in $\mathfrak{H}$ such that

$$
(B-\lambda)^{-1}-(A-\lambda)^{-1} \in \mathcal{F}_{n}(\mathfrak{H})
$$

holds for some (and hence for all) $\lambda \in \mathbb{C} \backslash \mathbb{R}$. Then the closed symmetric operator $S=A \cap B$ has finite deficiency indices $(n, n)$ in $\mathfrak{H}$ and there exists a boundary triplet $\Pi=\left\{\mathbb{C}^{n}, \Gamma_{0}, \Gamma_{1}\right\}$ for $S^{*}$ such that $A=\operatorname{ker}\left(\Gamma_{0}\right)$ and $B=\operatorname{ker}\left(\Gamma_{1}\right)$ holds. Moreover, if $\gamma(\cdot)$ and $M(\cdot)$ denote the $\gamma$-field and Weyl function of $\Pi$, then

$$
(B-\lambda)^{-1}-(A-\lambda)^{-1}=-\gamma(\lambda) M(\lambda)^{-1} \gamma(\bar{\lambda})^{*}
$$

holds for all $\lambda \in \rho(B) \cap \rho(A)$. 
Proof. According to Proposition 2.2 there is a boundary triplet $\Pi^{\prime}=$ $\left\{\mathbb{C}^{n}, \Gamma_{0}^{\prime}, \Gamma_{1}^{\prime}\right\}$ for $S^{*}$ such that $A=\operatorname{ker}\left(\Gamma_{0}^{\prime}\right)$ and $B=\operatorname{ker}\left(\Gamma_{1}^{\prime}-D \Gamma_{0}^{\prime}\right)$. Here the dissipative matrix $D$ is even symmetric since $B$ is selfadjoint. A simple calculation shows that $\Pi=\left\{\mathbb{C}^{n}, \Gamma_{0}, \Gamma_{1}\right\}$, where

$$
\Gamma_{0}:=\Gamma_{0}^{\prime} \quad \text { and } \quad \Gamma_{1}:=\Gamma_{1}-D \Gamma_{0},
$$

is also a boundary triplet for $S^{*}$. If $M(\cdot)$ is the Weyl function corresponding to the boundary triplet $\Pi^{\prime}$, then $\lambda \mapsto M(\lambda)-D$ is the Weyl function corresponding to the boundary triplet $\Pi$. This together with Proposition 2.2 and Corollary 2.4 implies the statement.

\section{A representation of the scattering matrix}

In this section we consider scattering systems $\{A, B\}$ consisting of two selfadjoint operators $A$ and $B$ in a separable Hilbert space $\mathfrak{H}$ and we assume that the difference of the resolvents of $A$ and $B$ is a finite rank operator, that is, for some $n \in \mathbb{N}$ we have

$$
(B-\lambda)^{-1}-(A-\lambda)^{-1} \in \mathcal{F}_{n}(\mathfrak{H})
$$

for one (and hence for all) $\lambda \in \rho(A) \cap \rho(B)$. Then the wave operators

$$
W_{ \pm}(B, A):=\mathrm{s}-\lim _{t \rightarrow \pm \infty} e^{i t B} e^{-i t A} P^{a c}(A)
$$

exist and are complete, where $P^{a c}(A)$ denotes the orthogonal projection onto the absolutely continuous subspace $\mathfrak{H}^{a c}(A)$ of $A$. Completeness means that the ranges of $W_{ \pm}(B, A)$ coincide with the absolutely continuous subspace $\mathfrak{H}^{a c}(B)$ of $B$; cf. [6, 19, 28, 29]. The scattering operator $S_{A B}$ of the scattering system $\{A, B\}$ is defined by

$$
S_{A B}:=W_{+}(B, A)^{*} W_{-}(B, A) .
$$

Since the scattering operator commutes with $A$ it follows that $S_{A B}$ is unitarily equivalent to a multiplication operator induced by a family $\left\{S_{A B}(\lambda)\right\}_{\lambda \in \mathbb{R}}$ of unitary operators in a spectral representation of $A^{a c}:=A\left\lceil\operatorname{dom}(A) \cap \mathfrak{H}^{a c}(A)\right.$. The aim of this section is to generalize a representation result of this so-called scattering matrix $\left\{S_{A B}(\lambda)\right\}_{\lambda \in \mathbb{R}}$ from [8].

According to (3.1) and Corollary 2.5 the (possibly nondensely defined) closed symmetric operator $S=A \cap B$ has deficiency indices $(n, n)$ and there exists a boundary triplet $\Pi=\left\{\mathbb{C}^{n}, \Gamma_{0}, \Gamma_{1}\right\}$ for $S^{*}$ such that $A=\operatorname{ker}\left(\Gamma_{0}\right)$ and $B=$ $\operatorname{ker}\left(\Gamma_{1}\right)$. The Weyl function $M(\cdot)$ corresponding to the boundary triplet $\Pi$ is a $\left[\mathbb{C}^{n}\right]$-valued Nevanlinna function. Therefore the limit

$$
M(\lambda):=M(\lambda+i 0)=\lim _{\varepsilon \rightarrow+0} M(\lambda+i \varepsilon)
$$

from the upper half-plane $\mathbb{C}_{+}$exists for a.e. $\lambda \in \mathbb{R}$; see [16, 17]. As $\Im$ m $(M(\lambda))$ is uniformly positive (uniformly negative) for all $\lambda \in \mathbb{C}_{+}\left(\lambda \in \mathbb{C}_{-}\right.$, respectively) the inverses $M(\lambda)^{-1}$ exist for all $\lambda \in \mathbb{C} \backslash \mathbb{R}$ and $-M(\cdot)^{-1}$ is also a $\left[\mathbb{C}^{n}\right]$-valued 
Nevanlinna function. Hence it follows that the $\operatorname{limit}_{\lim } \rightarrow 0+M(\lambda+i \varepsilon)^{-1}$ exists for a.e. $\lambda \in \mathbb{R}$ and coincides with the inverse of $M(\lambda)$ in (3.2) for a.e. $\lambda \in \mathbb{R}$.

In the following theorem we find a representation of the scattering matrix $\left\{S_{A B}(\lambda)\right\}_{\lambda \in \mathbb{R}}$ of the scattering system $\{A, B\}$ in the direct integral $L^{2}\left(\mathbb{R}, d \lambda, \mathcal{H}_{\lambda}\right)$, where

$$
\mathcal{H}_{\lambda}:=\operatorname{ran}(\Im \mathrm{m}(M(\lambda+i 0)) \text { for a.e. } \lambda \in \mathbb{R},
$$

cf. Appendix A. We will formulate and prove our result first for the case of a simple symmetric operator $S=A \cap B$ and discuss the general case afterwards in Theorem 3.2. For the special case that the simple symmetric operator $S=A \cap B$ is densely defined Theorem 3.1 reduces to [8, Theorem 3.8]. We remark that the proof of Theorem 3.1 differs from the proof of [8, Theorem 3.8]. Here we make use of the abstract representation result Theorem $\mathrm{A.2}$.

Theorem 3.1 Let $A$ and $B$ be selfadjoint operators in $\mathfrak{H}$ such that (3.1) is satisfied, suppose that the symmetric operator $S=A \cap B$ is simple and let $\Pi=\left\{\mathbb{C}^{n}, \Gamma_{0}, \Gamma_{1}\right\}$ be a boundary triplet for $S^{*}$ such that $A=\operatorname{ker}\left(\Gamma_{0}\right)$ and $B=$ $\operatorname{ker}\left(\Gamma_{1}\right)$; cf. Corollary 2.5. Let $M(\cdot)$ be the corresponding Weyl function and define the spaces $\mathcal{H}_{\lambda}$ for a.e. $\lambda \in \mathbb{R}$ as in (3.3).

Then $L^{2}\left(\mathbb{R}, d \lambda, \mathcal{H}_{\lambda}\right)$ performs a spectral representation of $A^{\text {ac }}$ such that the scattering matrix $\left\{S_{A B}(\lambda)\right\}_{\lambda \in \mathbb{R}}$ of the scattering system $\{A, B\}$ admits the representation

$$
S_{A B}(\lambda)=I_{\mathcal{H}_{\lambda}}-2 i \sqrt{\Im m(M(\lambda))} M(\lambda)^{-1} \sqrt{\Im m(M(\lambda))} \in\left[\mathcal{H}_{\lambda}\right]
$$

for a.e. $\lambda \in \mathbb{R}$, where $M(\lambda)=M(\lambda+i 0)$.

Proof. In order to verify the representation (3.4) of the scattering matrix $\left\{S_{A B}(\lambda)\right\}_{\lambda \in \mathbb{R}}$ we will make use of Theorem A.2. For this let us first rewrite the difference of the resolvents $(B-i)^{-1}$ and $(A-i)^{-1}$ as in (A.3). According to Corollary 2.5 we have

$$
(B-i)^{-1}-(A-i)^{-1}=-\gamma(i) M(i)^{-1} \gamma(-i)^{*} .
$$

Using (2.8) we find

$$
(B-i)^{-1}-(A-i)^{-1}=-(A+i)(A-i)^{-1} \gamma(-i) M(i)^{-1} \gamma(-i)^{*} .
$$

and hence the representation (A.3) follows if we set

$$
\phi(t):=\frac{t+i}{t-i}, \quad t \in \mathbb{R}, \quad C=\gamma(-i) \quad \text { and } \quad G=-M(i)^{-1} .
$$

Moreover, since $S$ is simple it follows from (2.2) that

$$
\mathfrak{H}=\operatorname{clospan}\left\{\operatorname{ker}\left(S^{*}-\lambda\right): \lambda \in \mathbb{C} \backslash \mathbb{R}\right\}
$$

holds. As $\operatorname{ran} C=\operatorname{ran} \gamma(-i)=\operatorname{ker}\left(S^{*}+i\right)$ one concludes in the same way as in the proof of [8, Lemma 3.4] that the condition

$$
\mathfrak{H}^{a c}(A)=\operatorname{clospan}\left\{E_{A}^{a c}(\delta) \operatorname{ran}(C): \delta \in \mathcal{B}(\mathbb{R})\right\}
$$

is satisfied. 
Next we express the $\left[\mathbb{C}^{n}\right]$-valued function

$$
\lambda \mapsto K(\lambda)=\frac{d}{d \lambda} C^{*} E_{A}((-\infty, \lambda)) C
$$

and its square root $\lambda \mapsto \sqrt{K(\lambda)}$ in terms of the Weyl function $M(\cdot)$ for a.e. $\lambda \in \mathbb{R}$. We have

$$
\begin{aligned}
K(\lambda) & =\lim _{\varepsilon \rightarrow+0} \frac{1}{2 \pi i} \gamma(-i)^{*}\left((A-\lambda-i \varepsilon)^{-1}-(A-\lambda+i \varepsilon)^{-1}\right) \gamma(-i) \\
& =\lim _{\varepsilon \rightarrow+0} \frac{\varepsilon}{\pi} \gamma(-i)^{*}(A-\lambda-i \varepsilon)^{-1}(A-\lambda+i \varepsilon)^{-1} \gamma(-i)
\end{aligned}
$$

and on the other hand by (2.10)

$$
\Im \mathrm{m}(M(\lambda+i \varepsilon))=\varepsilon \gamma(\lambda+i \varepsilon)^{*} \gamma(\lambda+i \varepsilon) .
$$

Inserting $\gamma(\lambda+i \varepsilon)=\left(I+(\lambda+i \varepsilon+i)(A-\lambda-i \varepsilon)^{-1} \gamma(-i)\right)$ (cf. (2.8) $)$ we obtain

$$
\Im \mathrm{m}(M(\lambda+i \varepsilon))=\varepsilon \gamma(-i)^{*}\left(I+A^{2}\right)(A-\lambda-i \varepsilon)^{-1}(A-\lambda+i \varepsilon)^{-1} \gamma(-i)
$$

and by comparing (3.7) and (3.8) we find

$$
\Im \mathrm{m}(M(\lambda))=\lim _{\varepsilon \rightarrow 0+} \Im \mathrm{m}(M(\lambda+i \varepsilon))=\pi\left(1+\lambda^{2}\right) K(\lambda)
$$

for a.e. $\lambda \in \mathbb{R}$. In particular, the finite-dimensional subspaces $\operatorname{ran}(K(\lambda))$ in Theorem A.2 coincide with the spaces $\mathcal{H}_{\lambda}=\operatorname{ran}(\Im \mathrm{m}(M(\lambda)))$ for a.e. $\lambda \in \mathbb{R}$ and therefore $L^{2}\left(\mathbb{R}, d \lambda, \mathcal{H}_{\lambda}\right)$ is a spectral representation of $A^{a c}$ and the scattering matrix $\left\{S_{A B}(\lambda)\right\}_{\lambda \in \mathbb{R}}$ admits the representation (A.4). Inserting the square root $\sqrt{K(\lambda)}$ from (3.9) into (A.4) we find

$$
S_{A B}(\lambda)=I_{\mathcal{H}_{\lambda}}+2 i\left(1+\lambda^{2}\right) \sqrt{\Im \mathrm{m}(M(\lambda))} Z(\lambda) \sqrt{\Im \mathrm{m}(M(\lambda))}
$$

and it remains to compute

$$
Z(\lambda)=\frac{1}{\lambda+i} Q^{*} Q+\frac{\phi(\lambda)}{(\lambda+i)^{2}} G+\lim _{\varepsilon \rightarrow 0+} Q^{*}(B-\lambda-i \varepsilon)^{-1} Q,
$$

where $Q=\phi(A) C G=-\gamma(i) M(i)^{-1}$, cf. (A.5), (3.6) and (3.5).

It follows from [8, Lemma 3.2] that

$$
Q^{*}(B-\lambda-i 0)^{-1} Q=\frac{1}{1+\lambda^{2}}\left(M(i)^{-1}-M(\lambda)^{-1}\right)+\frac{1}{\lambda+i} \Im \mathrm{m}\left(M(i)^{-1}\right)
$$

holds for a.e. $\lambda \in \mathbb{R}$ and from (2.10) we obtain

$$
\begin{aligned}
Q^{*} Q & =\left(M(i)^{-1}\right)^{*} \gamma(i)^{*} \gamma(i) M(i)^{-1}=\left(M(i)^{-1}\right)^{*} \Im \mathrm{m}(M(i)) M(i)^{-1} \\
& =-\Im \mathrm{m}\left(M(i)^{-1}\right) .
\end{aligned}
$$

Therefore we conclude from (3.13) and (3.6) that (3.11) takes the form

$$
Z(\lambda)=-\frac{1}{\lambda+i} \Im \mathrm{m}\left(M(i)^{-1}\right)-\frac{1}{1+\lambda^{2}} M(i)^{-1}+Q^{*}(B-\lambda-i 0)^{-1} Q
$$


and by inserting (3.12) we find $Z(\lambda)=-\left(1+\lambda^{2}\right)^{-1} M(\lambda)^{-1}$. Hence (3.10) turns into the representation (3.4) of the scattering matrix $\left\{S_{A B}(\lambda)\right\}$.

In general it may happen that the operator $S=A \cap B$ is not simple, that is, there is a nontrivial decomposition of the Hilbert space $\mathfrak{H}=\widehat{\mathfrak{H}} \oplus \mathfrak{K}$ such that

$$
S=\widehat{S} \oplus H,
$$

where $\widehat{S}$ is simple symmetric operator in $\widehat{\mathfrak{H}}$ and $H$ is a selfadjoint operator in $\mathfrak{K}$, cf. Section 2.1. Then there exist selfadjoint extensions $\widehat{A}$ and $\widehat{B}$ of $\widehat{S}$ in $\widehat{\mathfrak{H}}$ such that

$$
A=\widehat{A} \oplus H \quad \text { and } \quad B=\widehat{B} \oplus H .
$$

The next result extends the representation of the scattering matrix in Theorem 3.1 to the case of a non-simple $S$.

Theorem 3.2 Let $A$ and $B$ be selfadjoint operators in $\mathfrak{H}$ such that (3.1) is satisfied, let $S=A \cap B$ be decomposed as in (3.14) and let $\Pi=\left\{\mathbb{C}^{n}, \Gamma_{0}, \Gamma_{1}\right\}$ be a boundary triplet for $S^{*}$ such that $A=\operatorname{ker}\left(\Gamma_{0}\right)$ and $B=\operatorname{ker}\left(\Gamma_{1}\right) ; c f$. Corollary 2.5. Furthermore, let $L^{2}\left(\mathbb{R}, d \lambda, \mathcal{K}_{\lambda}\right)$ be a spectral representation of the absolutely continuous part $H^{a c}$ of the selfadjoint operator $H$ in the Hilbert space $\mathfrak{K}$.

Then $L^{2}\left(\mathbb{R}, d \lambda, \mathcal{H}_{\lambda} \oplus \mathcal{K}_{\lambda}\right)$ is a spectral representation of $A^{a c}$ and the scattering matrix $\left\{S_{A B}(\lambda)\right\}_{\lambda \in \mathbb{R}}$ is given by

$$
S_{A B}(\lambda)=\left(\begin{array}{cc}
S_{\widehat{A}, \widehat{B}}(\lambda) & 0 \\
0 & I_{\mathcal{K}_{\lambda}}
\end{array}\right) \in\left[\mathcal{H}_{\lambda} \oplus \mathcal{K}_{\lambda}\right]
$$

for a.e. $\lambda \in \mathbb{R}$, where $\mathcal{H}_{\lambda}=\operatorname{ran}(\Im \mathrm{m}(M(\lambda+i 0))), M(\cdot)$ is the Weyl function corresponding to the boundary triplet $\Pi$ and

$$
S_{\widehat{A} \widehat{B}}(\lambda)=I_{\mathcal{H}_{\lambda}}-2 i \sqrt{\Im \mathrm{m}(M(\lambda))} M(\lambda)^{-1} \sqrt{\Im \mathrm{m}(M(\lambda))} \in\left[\mathcal{H}_{\lambda}\right]
$$

is the scattering matrix of the scattering system $\{\widehat{A}, \widehat{B}\}$ from (3.15).

Proof. It follows from the decomposition (3.15) that the absolutely continuous subspaces $\mathfrak{H}^{a c}(A)$ and $\mathfrak{H}^{a c}(B)$ can be written as the orthogonal sums

$$
\mathfrak{H}^{a c}(A)=\widehat{\mathfrak{H}}^{a c}(\widehat{A}) \oplus \mathfrak{K}^{a c}(H) \quad \text { and } \quad \mathfrak{H}^{a c}(B)=\widehat{\mathfrak{H}}^{a c}(\widehat{B}) \oplus \mathfrak{K}^{a c}(H)
$$

of the absolutely continuous subspaces of $\widehat{A}$ and $\widehat{B}$, and the absolutely continuous subspace $\mathfrak{K}^{a c}(H)$ of the selfadjoint operator $H$ in $\mathfrak{K}$. Therefore the wave operators of $W_{ \pm}(B, A)$ of the scattering system $\{A, B\}$ can be written with the wave operators $W_{ \pm}(\widehat{B}, \widehat{A})$ of the scattering system $\{\widehat{A}, \widehat{B}\}$ in the form

$$
W_{ \pm}(B, A)=W_{ \pm}(\widehat{B}, \widehat{A}) \oplus I_{\mathfrak{K}^{a c}}(H) .
$$

This implies the corresponding decomposition of the scattering operator $S_{A B}$ in $S=S_{\widehat{A} \widehat{B}} \oplus I_{\mathfrak{K}^{a c}(H)}$ and hence the scattering matrix $\left\{S_{A B}(\lambda)\right\}_{\lambda \in \mathbb{R}}$ of the scattering system $\{A, B\}$ coincides with the orthogonal sum of the scattering matrix $\left\{S_{\widehat{A} \widehat{B}}(\lambda)\right\}_{\lambda \in \mathbb{R}}$ of the scattering system $\{\widehat{A}, \widehat{B}\}$ and the identity operator in the spectral representation $L^{2}\left(\mathbb{R}, d \lambda, \mathcal{K}_{\lambda}\right)$ of $H^{a c}$. 
It is not difficult to see that $\widehat{\Pi}=\left\{\mathbb{C}^{n}, \widehat{\Gamma}_{0}, \widehat{\Gamma}_{1}\right\}$, where $\widehat{\Gamma}_{0}$ and $\widehat{\Gamma}_{1}$ denote the restrictions of the boundary mappings $\Gamma_{0}$ and $\Gamma_{1}$ from $S^{*}=(\widehat{S})^{*} \oplus H$ onto $(\widehat{S})^{*}$, is a boundary triplet for $(\widehat{S})^{*}$ such that $\widehat{A}=\operatorname{ker}\left(\widehat{\Gamma}_{0}\right)$ and $\widehat{B}=\operatorname{ker}\left(\widehat{\Gamma}_{1}\right)$. Moreover, the Weyl function corresponding to $\widehat{\Pi}$ coincides with the Weyl function $M(\cdot)$ corresponding to $\Pi$. Hence, by Theorem 3.1 the scattering matrix $\left\{S_{\widehat{A} \widehat{B}}(\lambda)\right\}_{\lambda \in \mathbb{R}}$ is given by (3.4).

\section{Dissipative and Lax-Phillips scattering sys- tems}

In this section we consider a scattering systems $\{A, B\}$ consisting of a selfadjoint operator $A$ and a maximal dissipative operator $B$ in the Hilbert space $\mathfrak{H}$. As above it is assumed that

$$
(B-\lambda)^{-1}-(A-\lambda)^{-1} \in \mathcal{F}_{n}(\mathfrak{H}), \quad \lambda \in \rho(A) \cap \rho(B),
$$

holds for some $n \in \mathbb{N}$. Then the closed symmetric operator $S=A \cap B$ is in general not densely defined and its deficiency indices are $(n, n)$. By Corollary 2.4 there exists a boundary triplet $\Pi=\left\{\mathbb{C}^{n}, \Gamma_{0}, \Gamma_{1}\right\}$ for $S^{*}$ and a dissipative $n \times n$ matrix $D$ such that $A=\operatorname{ker}\left(\Gamma_{0}\right), B=\operatorname{ker}\left(\Gamma_{1}-D \Gamma_{0}\right)$ and

$$
(B-\lambda)^{-1}-(A-\lambda)^{-1}=\gamma(\lambda)(D-M(\lambda))^{-1} \gamma(\bar{\lambda})^{*}
$$

holds. For our later purposes in Section 5 it is sufficient to investigate the special case $\operatorname{ker}(\Im m(D))=\{0\}$, the general case can be treated in the same way as in [7, 9].

For the investigation of the dissipative scattering system $\{A, B\}$ it is useful to construct a so-called minimal selfadjoint dilation $L$ of the maximal dissipative operator $B$. For the explicit construction of $L$ we will use the following lemma which also shows how the constant function $\mathbb{C}_{+} \ni \lambda \mapsto-i \Im \mathrm{m}(D), \lambda \in \mathbb{C}_{+}$, can be realized as a Weyl function. A detailed proof of Lemma 4.1 can be found in 7].

Lemma 4.1 Let $T$ be the first order differential operator in the Hilbert space $L^{2}\left(\mathbb{R}, \mathbb{C}^{n}\right)$ defined by

$$
(T g)(x)=-i g^{\prime}(x), \quad \operatorname{dom}(T)=\left\{g \in W_{2}^{1}\left(\mathbb{R}, \mathbb{C}^{n}\right): g(0)=0\right\} .
$$

Then the following holds.

(i) $T$ is a densely defined closed simple symmetric operator with deficiency indices $(n, n)$.

(ii) The adjoint operator is

$$
\left(T^{*} g\right)(x)=-i g^{\prime}(x), \quad \operatorname{dom}\left(T^{*}\right)=W_{2}^{1}\left(\mathbb{R}_{-}, \mathbb{C}^{n}\right) \oplus W_{2}^{1}\left(\mathbb{R}_{+}, \mathbb{C}^{n}\right) .
$$

(iii) The triplet $\Pi_{T}=\left\{\mathbb{C}^{n}, \Upsilon_{0}, \Upsilon_{1}\right\}$, where

$$
\begin{aligned}
& \Upsilon_{0} \hat{g}:=\frac{1}{\sqrt{2}}(-\Im \mathrm{m}(D))^{-\frac{1}{2}}(g(0+)-g(0-)), \\
& \Upsilon_{1} \hat{g}:=\frac{i}{\sqrt{2}}(-\Im \mathrm{m}(D))^{\frac{1}{2}}(g(0+)+g(0-)), \quad \hat{g}=\left\{g, T^{*} g\right\},
\end{aligned}
$$


is a boundary triplet for $T^{*}$ and $T_{0}=\operatorname{ker}\left(\Upsilon_{0}\right)$ is the selfadjoint first order differential operator in $L^{2}\left(\mathbb{R}, \mathbb{C}^{n}\right)$ defined on $W_{2}^{1}\left(\mathbb{R}, \mathbb{C}^{n}\right)$.

(iv) The Weyl function $\tau(\cdot)$ corresponding to the boundary triplet $\Pi_{T}=$ $\left\{\mathbb{C}^{n}, \Upsilon_{0}, \Upsilon_{1}\right\}$ is given by

$$
\tau(\lambda)= \begin{cases}-i \Im \mathrm{m}(D), & \lambda \in \mathbb{C}_{+}, \\ i \Im \mathrm{m}(D), & \lambda \in \mathbb{C}_{-}\end{cases}
$$

Let $S=A \cap B$ and let $T$ be the first order differential operator from Lemma 4.1. It is clear that

$$
\left(\begin{array}{ll}
S & 0 \\
0 & T
\end{array}\right)
$$

is a closed symmetric operator in the Hilbert space $\mathfrak{H} \oplus L^{2}\left(\mathbb{R}, \mathbb{C}^{n}\right)$ with deficiency indices $(2 n, 2 n)$ and the adjoint of (4.2) is the orthogonal sum of the relation $S^{*}$ and the operator $T^{*}$ from Lemma 4.1. The next theorem, which is a variant of [7. Theorem 3.2], shows how a minimal selfadjoint dilation of the dissipative operator $B=\operatorname{ker}\left(\Gamma_{1}-D \Gamma_{0}\right)$ can be constructed. For the particular case of Sturm-Liouville operators with dissipative boundary conditions this construction goes back to B.S. Pavlov; cf. [25, 26].

Theorem 4.2 Let $A$ be a selfadjoint operator and let $B$ be a maximal dissipative operator in $\mathfrak{H}$ such that (4.1) holds. Let $\Pi=\left\{\mathbb{C}^{n}, \Gamma_{0}, \Gamma_{1}\right\}$ be a boundary triplet for $S^{*}, S=A \cap B$, and let $D$ be a dissipative $n \times n$-matrix with $\operatorname{ker}(\Im \mathrm{m}(D))=$ $\{0\}$ such that $A=\operatorname{ker}\left(\Gamma_{0}\right)$ and $B=\operatorname{ker}\left(\Gamma_{1}-D \Gamma_{0}\right)$; cf. Proposition [2.2. If $\Pi_{T}=\left\{\mathbb{C}^{n}, \Upsilon_{0}, \Upsilon_{1}\right\}$ is the boundary triplet of $T^{*}$ introduced in Lemma 4.1. then the operator

$$
L\left(\begin{array}{l}
f \\
g
\end{array}\right)=\left(\begin{array}{c}
f^{\prime} \\
T^{*} g
\end{array}\right), \quad \hat{f}=\left\{f, f^{\prime}\right\} \in S^{*}, \hat{g}=\left\{g, T^{*} g\right\},
$$

with domain

$$
\operatorname{dom}(L)=\left\{\left(\begin{array}{l}
f \\
g
\end{array}\right) \in \operatorname{dom}\left(S^{*}\right) \oplus \operatorname{dom}\left(T^{*}\right): \begin{array}{c}
\Gamma_{0} \hat{f}-\Upsilon_{0} \hat{g}=0 \\
\left(\Gamma_{1}-\Re \mathrm{e}(D) \Gamma_{0}\right) \hat{f}=-\Upsilon_{1} \hat{g}
\end{array}\right\}
$$

is a minimal selfadjoint dilation of the maximal dissipative operator $B$, that is, for all $\lambda \in \mathbb{C}_{+}$

$$
P_{\mathfrak{H}}(L-\lambda)^{-1} \Gamma_{\mathfrak{H}}=(B-\lambda)^{-1}
$$

holds and the condition $\mathfrak{H} \oplus L^{2}\left(\mathbb{R}, \mathbb{C}^{n}\right)=\operatorname{clospan}\left\{(L-\lambda)^{-1} \mathfrak{H}: \lambda \in \mathbb{C} \backslash \mathbb{R}\right\}$ is satisfied.

Proof. Besides the assertion that $L$ is an operator the proof of Theorem 4.2 is essentially the same as the proof of [7, Theorem 3.2]. The fact that the restriction $L$ of the relation $S^{*} \oplus T^{*}$ is an operator can be seen as follows: Suppose that $\hat{f} \oplus \hat{g} \in L$, where $\left\{0, f^{\prime}\right\} \in S^{*},\left\{0, g^{\prime}\right\} \in T^{*}$. Since $T^{*}$ is an operator we have $g^{\prime}=0$ and this implies $\hat{g}=0$. Therefore we obtain from the boundary conditions in $\operatorname{dom}(L)$ that

$$
\Gamma_{0} \hat{f}=\Upsilon_{0} \hat{g}=0
$$


holds. Hence $\hat{f}=\left\{f, f^{\prime}\right\}$ belongs to $A=\operatorname{ker}\left(\Gamma_{0}\right)$ which is an operator. Therefore $f^{\prime}=0$ and $L$ is an operator.

Let $L$ be the minimal selfadjoint dilation of the maximal dissipative operator $B$ from Theorem 4.2 and define a selfadjoint operator $K$ in $\mathfrak{H} \oplus L^{2}\left(\mathbb{R}, \mathbb{C}^{n}\right)$ by

$$
K:=\left(\begin{array}{cc}
A & 0 \\
0 & T_{0}
\end{array}\right)
$$

where $T_{0}=\operatorname{ker}\left(\Upsilon_{0}\right)$ is the selfadjoint first order differential operator from Lemma 4.1. In the following theorem we consider the scattering system $\{K, L\}$ in the Hilbert space $\mathfrak{H} \oplus L^{2}\left(\mathbb{R}, \mathbb{C}^{n}\right)$. The operator $R:=K \cap L$ is symmetric and may have a nontrivial selfadjoint part $H$ which acts in the Hilbert space

$$
\left(\mathfrak{H} \oplus L^{2}\left(\mathbb{R}, \mathbb{C}^{n}\right)\right) \ominus \operatorname{clospan}\left\{\operatorname{ker}\left(R^{*}-\lambda\right): \lambda \in \mathbb{C} \backslash \mathbb{R}\right\} .
$$

Hence the operators $K$ and $L$ admit the decompositions

$$
K=\widehat{K} \oplus H \quad \text { and } \quad L=\widehat{L} \oplus H,
$$

with selfadjoint operators $\widehat{K}$ and $\widehat{L}$ in clospan $\left\{\operatorname{ker}\left(R^{*}-\lambda\right): \lambda \in \mathbb{C} \backslash \mathbb{R}\right\}$ and we have $R=\widehat{R} \oplus H$, where $\widehat{R}=\widehat{K} \cap \widehat{L}$. In particular, $\widehat{K}$ and $\widehat{L}$ are both selfadjoint extensions of the closed simple symmetric operator $\widehat{R}$. We remark that the symmetric operator $R$ is an $n$-dimensional extension of the orthogonal sum in (4.2); this follows easily from the next theorem. In the following we assume that $L^{2}\left(\mathbb{R}, d \lambda, \mathcal{K}_{\lambda}\right)$ is a spectral representation of the absolutely continuous part $H^{a c}$ of $H$.

Theorem 4.3 Let $A$ be a selfadjoint operator and let $B$ be a maximal dissipative operator in $\mathfrak{H}$ such that (4.1) holds. Let $\Pi=\left\{\mathbb{C}^{n}, \Gamma_{0}, \Gamma_{1}\right\}$ be a boundary triplet for $S^{*}, S=A \cap B$, and let $D$ be a dissipative $n \times n$-matrix with $\operatorname{ker}(\Im \mathrm{m}(D))=$ $\{0\}$ such that $A=\operatorname{ker}\left(\Gamma_{0}\right)$ and $B=\operatorname{ker}\left(\Gamma_{1}-D \Gamma_{0}\right) ; c f$. Proposition 2.2. If $L$ is the minimal self-adjoint dilation of $B$ in Theorem 4.2 and $K$ is given by (4.3), then

$$
(K-\lambda)-(L-\lambda)^{-1} \in \mathcal{F}_{n}, \quad \lambda \in \mathbb{C} \backslash \mathbb{R} .
$$

Moreover, if $L^{2}\left(\mathbb{R}, d \lambda, \mathcal{K}_{\lambda}\right)$ is a spectral representation of $H^{a c}$, where $H$ is the maximal self-adjoint part of $R=K \cap L$, then $L^{2}\left(\mathbb{R}, d \lambda, \mathbb{C}^{n} \oplus \mathcal{K}_{\lambda}\right)$ is a spectral representation of $K$ and the scattering matrix $\left\{S_{K L}(\lambda)\right\}_{\lambda \in \mathbb{R}}$ of the scattering system $\{K, L\}$ admits the representation

$$
S_{K L}(\lambda)=\left(\begin{array}{cc}
S_{\widehat{K} \widehat{L}}(\lambda) & 0 \\
0 & I_{\mathcal{K}_{\lambda}}
\end{array}\right) \in\left[\mathbb{C}^{n} \oplus \mathcal{K}_{\lambda}\right]
$$

for a.e. $\lambda \in \mathbb{R}$, where

$$
S_{\widehat{K} \widehat{L}}(\lambda)=I_{\mathbb{C}^{n}}-2 i \sqrt{\Im \mathrm{m}(M(\lambda)-D)}(M(\lambda)-D)^{-1} \sqrt{\Im \mathrm{m}(M(\lambda)-D)}
$$

is the scattering matrix of the scattering system $\{\widehat{K}, \widehat{L}\}, M(\cdot)$ is the Weyl function of the boundary triplet $\Pi$ and $M(\lambda)=M(\lambda+i 0)$. 
Proof. We are going to apply Theorem 3.2 to the scattering system $\{K, L\}$. For this we consider the symmetric operator $R=K \cap L$ and note that the operator $K$ is given by $\operatorname{ker}\left(\Gamma_{0}\right) \oplus \operatorname{ker}\left(\Upsilon_{0}\right)$. Hence the boundary condition $\Gamma_{0} \hat{f}-\Upsilon_{0} \hat{g}=0$ in $\operatorname{dom}(L)$ is automatically fulfilled and this implies that the intersection $R=$ $K \cap L$ given by

$$
\begin{aligned}
R\left(\begin{array}{l}
f \\
g
\end{array}\right) & =K\left(\begin{array}{l}
f \\
g
\end{array}\right)=L\left(\begin{array}{l}
f \\
g
\end{array}\right) \\
\operatorname{dom}(R) & =\left\{\left(\begin{array}{l}
f \\
g
\end{array}\right) \in \operatorname{dom} K:\left(\Gamma_{1}-\Re \mathrm{e}(D) \Gamma_{0}\right) \hat{f}=-\Upsilon_{1} \hat{g}\right\},
\end{aligned}
$$

where $\hat{f}=\{f, A f\}$ and $\hat{g}=\left\{g, T_{0} g\right\}$. It is not difficult to verify that the adjoint operator $R^{*}$ has the form

$$
R^{*}=\left\{\hat{f} \oplus \hat{g} \in S^{*} \oplus T^{*}: \Gamma_{0} \hat{f}=\Upsilon_{0} \hat{g}\right\}
$$

and $\widetilde{\Pi}=\left\{\mathbb{C}^{n}, \widetilde{\Gamma}_{0}, \widetilde{\Gamma}_{1}\right\}$, where

$$
\widetilde{\Gamma}_{0}(\hat{f} \oplus \hat{g})=\Gamma_{0} \hat{f} \quad \text { and } \quad \widetilde{\Gamma}_{1}(\hat{f} \oplus \hat{g})=\left(\Gamma_{1}-\Re \mathrm{e}(D) \Gamma_{0}\right) \hat{f}+\Upsilon_{1} \hat{g}
$$

is a boundary triplet for $R^{*}$. Observe that $K=\operatorname{ker}\left(\widetilde{\Gamma}_{0}\right)$ and $L=\operatorname{ker}\left(\widetilde{\Gamma}_{1}\right)$. This also implies that the difference of the resolvents of $K$ and $L$ in (4.4) is a rank $n$ operator; cf. Corollary 2.5.

Let us compute the Weyl function $\widetilde{M}$ corresponding to the boundary triplet $\widetilde{\Pi}$. For $\lambda \in \mathbb{C}_{+}$and $\hat{f} \oplus \hat{g} \in \widehat{\mathcal{N}}_{\lambda, R^{*}}$ we have $\hat{f} \in \widehat{\mathcal{N}}_{\lambda, S^{*}}, \hat{g} \in \widehat{\mathcal{N}}_{\lambda, T^{*}}$ and $\Gamma_{0} \hat{f}=\Upsilon_{0} \hat{g}$. Hence the definition of the Weyl function and Lemma 4.1 imply

$$
\begin{aligned}
\widetilde{M}(\lambda) \widetilde{\Gamma}_{0}(\hat{f} \oplus \hat{g}) & =\widetilde{\Gamma}_{1}(\hat{f} \oplus \hat{g})=\Gamma_{1} \hat{f}-\Re \mathrm{e}(D) \Gamma_{0} \hat{f}+\Upsilon_{1} \hat{g} \\
& =M(\lambda) \Gamma_{0} \hat{f}-\Re \mathrm{e}(D) \Gamma_{0} \hat{f}-i \Im \mathrm{m}(D) \Upsilon_{0} \hat{g} \\
& =(M(\lambda)-D) \Gamma_{0} \hat{f}=(M(\lambda)-D) \widetilde{\Gamma}_{0}(\hat{f} \oplus \hat{g})
\end{aligned}
$$

and therefore $\widetilde{M}(\lambda)=M(\lambda)-D$ for $\lambda \in \mathbb{C}_{+}$. As $D$ is a dissipative matrix and $\operatorname{ker}(\Im \mathrm{m}(D))=\{0\}$ by assumption it follows that

$$
\Im \mathrm{m}(\widetilde{M}(\lambda+i 0))=\Im \mathrm{m}(M(\lambda+i 0))-\Im \mathrm{m}(D)
$$

is uniformly positive and hence $\operatorname{ran}(\Im \mathrm{m}(\widetilde{M}(\lambda+i 0)))=\mathbb{C}^{n}$. Now Theorem 3.2 applied to the boundary triplet $\widetilde{\Pi}$ and the corresponding Weyl function $\widetilde{M}$ yields the statement of Theorem 4.3 .

For our later purposes it is useful to express the scattering matrix $\left\{S_{K L}(\lambda)\right\}_{\lambda \in \mathbb{R}}$ in Theorem 4.3 in a slightly different form. The following proposition extends [7. Theorem 3.6] to the case where $S=A \cap B$ is not necessarily densely defined. The proof is almost the same and will not be repeated.

Proposition 4.4 Let the assumptions of Theorem 4.3 be satisfied, assume, in addition, that $S=A \cap B$ is simple and let $L^{2}\left(\mathbb{R}, d \lambda, \mathcal{H}_{\lambda}\right), \mathcal{H}_{\lambda}=$ $\operatorname{ran}(\Im \mathrm{m}(M(\lambda))), M(\lambda):=M(\lambda+i 0)$, be a spectral representation of $A^{a c}$. 
Then $L^{2}\left(\mathbb{R}, d \lambda, \mathcal{H}_{\lambda} \oplus \mathbb{C}^{n}\right)$ is a spectral representation of $K^{a c}=A^{a c} \oplus T_{0}$ such that the scattering matrix $\left\{S_{K L}(\lambda)\right\}_{\lambda \in \mathbb{R}}$ of the scattering system $\{K, L\}$ can be expressed by

$$
S_{K L}(\lambda)=\left(\begin{array}{cc}
I_{\mathcal{H}_{\lambda}} & 0 \\
0 & I_{\mathbb{C}^{n}}
\end{array}\right)+2 i\left(\begin{array}{cc}
S_{11}(\lambda) & S_{12}(\lambda) \\
S_{21}(\lambda) & S_{22}(\lambda)
\end{array}\right) \in\left[\mathcal{H}_{\lambda} \oplus \mathbb{C}^{n}\right]
$$

for a.e. $\lambda \in \mathbb{R}$, where

$$
\begin{aligned}
& S_{11}(\lambda)=\sqrt{\Im \mathrm{m}(M(\lambda))}(D-M(\lambda))^{-1} \sqrt{\Im \mathrm{m}(M(\lambda))}, \\
& S_{12}(\lambda)=\sqrt{\Im \mathrm{m}(M(\lambda))}(D-M(\lambda))^{-1} \sqrt{-\Im \mathrm{m}(D)}, \\
& S_{21}(\lambda)=\sqrt{-\Im \mathrm{m}(D)}(D-M(\lambda))^{-1} \sqrt{-\Im \mathrm{m}(M(\lambda))}, \\
& S_{22}(\lambda)=\sqrt{-\Im \mathrm{m}(D)}(D-M(\lambda))^{-1} \sqrt{-\Im \mathrm{m}(D)} .
\end{aligned}
$$

Remark 4.5 If $S=A \cap B$ is simple we find by combining Theorem 4.3 with Proposition 4.4 that $\operatorname{dim}\left(\mathcal{K}_{\lambda}\right)=\operatorname{dim}\left(\mathcal{H}_{\lambda}\right)$ holds for a.e. $\lambda \in \mathbb{R}$, i.e., the spectral multiplicity of $H^{a c}$, where $H$ is the maximal self-adjoint part of $R=K \cap L$ is equal to the spectral multiplicity of $A^{a c}$.

In the following we are going to interpret the right lower corner $I+2 i S_{22}$ of the scattering matrix $\left\{S_{K L}(\lambda)\right\}_{\lambda \in \mathbb{R}}$ in Proposition 4.4 as the scattering matrix corresponding to a Lax-Phillips scattering system; see, e.g., 6, 22 for further details. For this purpose we decompose $L^{2}\left(\mathbb{R}, \mathbb{C}^{n}\right)$ into the orthogonal sum of the subspaces

$$
\mathcal{D}_{-}:=L^{2}\left(\mathbb{R}_{-}, \mathbb{C}^{n}\right) \text { and } \quad \mathcal{D}_{+}:=L^{2}\left(\mathbb{R}_{+}, \mathbb{C}^{n}\right),
$$

and denote the natural embeddings of $\mathcal{D}_{ \pm}$into $\mathfrak{H} \oplus L^{2}\left(\mathbb{R}, \mathbb{C}^{n}\right)$ by $J_{ \pm}$. The subspaces $\mathcal{D}_{+}$and $\mathcal{D}_{-}$are called outgoing and incoming subspaces, respectively, for the selfadjoint dilation $L$ in $\mathfrak{H} \oplus L^{2}\left(\mathbb{R}, \mathbb{C}^{n}\right)$, i.e.

$$
e^{-i t L} \mathcal{D}_{ \pm} \subseteq \mathcal{D}_{ \pm}, \quad t \in \mathbb{R}_{ \pm}, \quad \text { and } \bigcap_{t \in \mathbb{R}} e^{-i t L} \mathcal{D}_{ \pm}=\{0\}
$$

hold. The system $\left\{L, \mathcal{D}_{-}, \mathcal{D}_{+}\right\}$is called Lax-Phillips scattering system and the corresponding Lax-Phillips wave operators are defined by

$$
\Omega_{ \pm}:=\mathrm{s}_{t \rightarrow \pm \infty} \lim _{t \rightarrow \pm} e^{i t L} J_{ \pm} e^{-i t T_{0}}: L^{2}\left(\mathbb{R}, \mathbb{C}^{n}\right) \rightarrow \mathfrak{H} \oplus L^{2}\left(\mathbb{R}, \mathbb{C}^{n}\right)
$$

cf. 6]. Since $\mathrm{s}-\lim _{t \rightarrow \pm \infty} J_{\mp} e^{-i t T_{0}}=0$ the restrictions of the wave operators $W_{ \pm}(L, K)$ of the scattering system $\{K, L\}$ onto $L^{2}\left(\mathbb{R}, \mathbb{C}^{n}\right)$ coincide with the Lax-Phillips wave operators $\Omega_{ \pm}$and hence the Lax-Phillips scattering operator $S^{L P}:=\Omega_{+}^{*} \Omega_{-}$is given by $S^{L P}=P_{L^{2}} S_{K L} \iota_{L^{2}}$, where $S_{K L}$ is the scattering operator of the scattering system $\{K, L\}, P_{L^{2}}$ is the orthogonal projection from $\mathfrak{H} \oplus L^{2}\left(\mathbb{R}, \mathbb{C}^{n}\right)$ onto $L^{2}\left(\mathbb{R}, \mathbb{C}^{n}\right)$ and $\iota_{L^{2}}$ denotes the canonical embedding. Hence the Lax-Phillips scattering operator $S^{L P}$ is a contraction in $L^{2}\left(\mathbb{R}, \mathbb{C}^{n}\right)$ and commutes with the selfadjoint differential operator $T_{0}$. Therefore $S^{L P}$ is unitarily equivalent to a multiplication operator induced by a family $\left\{S^{L P}(\lambda)\right\}_{\lambda \in \mathbb{R}}$ of contractive operators in $L^{2}\left(\mathbb{R}, \mathbb{C}^{n}\right)$; this family is called the Lax-Phillips scattering matrix.

The above considerations together with Proposition 4.4 immediately imply the following corollary on the representation of the Lax-Phillips scattering matrix; cf. [7, Corollary 3.10]. 
Corollary 4.6 Let the assumptions of Proposition 4.4 be satisfied. If $\left\{L, \mathcal{D}_{-}, \mathcal{D}_{+}\right\}$is the Lax-Phillips scattering system from above, then the LaxPhillips scattering matrix $\left\{S^{L P}(\lambda)\right\}_{\lambda \in \mathbb{R}}$ admits the representation

$$
S^{L P}(\lambda)=I_{\mathbb{C}^{n}}+2 i \sqrt{-\Im \mathrm{m}(D)}(D-M(\lambda))^{-1} \sqrt{-\Im \mathrm{m}(D)}
$$

for a.e. $\lambda \in \mathbb{R}$, where $M(\lambda)=M(\lambda+i 0)$.

We mention that Corollary 4.6 also implies a well-known result of Adamyan and Arov in [1, 2, 3, 4] on the relation of the Lax-Phillips scattering matrix with the characteristic function of the maximal dissipative operator $B$; see [7] for further details.

\section{An inverse scattering problem}

Let $W: \mathbb{C}_{+} \rightarrow\left[\mathbb{C}^{n}\right]$ be a contractive analytic matrix function defined on the upper half-plane $\mathbb{C}_{+}$. Then the limit

$$
W(\lambda)=W(\lambda+i 0)=\lim _{y \rightarrow+0} W(\lambda+i y)
$$

exists for a.e. $\lambda \in \mathbb{R}$. In the following theorem we show that under some mild additional conditions the limit of the function $W$ can be regarded as the scattering matrix of a Lax-Phillips scattering system $\left\{L, \mathcal{D}_{-}, \mathcal{D}_{+}\right\}$, where $L$ is the minimal selfadjoint dilation of some maximal dissipative operator in a Hilbert space $\mathfrak{H}$ as in the previous section.

Theorem 5.1 Let $W: \mathbb{C}_{+} \rightarrow\left[\mathbb{C}^{n}\right]$ be a contractive analytic function such that the conditions

$$
\operatorname{ker}\left(I-W(\eta)^{*} W(\eta)\right)=\{0\}, \quad \eta \in \mathbb{C}_{+},
$$

and

$$
\lim _{y \rightarrow+\infty} \frac{1}{y}(I-W(i y))^{-1}=0
$$

are satisfied. Then the following holds:

(i) There exists a separable Hilbert space $\mathfrak{H}$, a (in general nondensely defined) simple symmetric operator $S$ with deficiency indices $(n, n)$ in $\mathfrak{H}$, a boundary triplet $\Pi=\left\{\mathbb{C}^{n}, \Gamma_{0}, \Gamma_{1}\right\}$ for $S^{*}$ with Weyl function $M(\cdot)$ and a dissipative matrix $D \in\left[\mathbb{C}^{n}\right]$ with $\operatorname{ker}(\Im \mathrm{m}(D))=\{0\}$ such that $W(\cdot)$ admits the representation

$$
W(\mu)=I+2 i \sqrt{-\Im m(D)}(D-M(\mu))^{-1} \sqrt{-\Im m(D)}
$$

for all $\mu \in \mathbb{C}_{+}$and a.e. $\mu \in \mathbb{R}$, where $W(\mu)=W(\mu+i 0)$ and $M(\mu)=$ $M(\mu+i 0)$.

(ii) The function $\mathbb{R} \ni \mu \mapsto W(\mu)$ is the Lax-Phillips scattering matrix of the Lax-Phillips scattering system $\left\{L, \mathcal{D}_{-}, \mathcal{D}_{+}\right\}$, where $L$ is the minimal selfadjoint dilation of the maximal dissipative extension $B=\operatorname{ker}\left(\Gamma_{1}-D \Gamma_{0}\right)$ of $S$ in Theorem 4.2 and the incoming and outgoing subspaces are $\mathcal{D}_{ \pm}=$ $L^{2}\left(\mathbb{R}_{ \pm}, \mathbb{C}^{n}\right)$. 
(iii) If, in addition, the condition

$$
\lim _{y \rightarrow+\infty} y^{1 / 2}\left\|\sqrt{I-W(i y)^{*} W(i y)}(I-W(i y))^{-1} h\right\|=\infty
$$

holds for all $h \in \mathcal{H}, h \neq 0$, then the symmetric operator $S$ is densely defined.

(iv) If $W(\cdot)$ is an inner function, i.e. $W(\mu+i 0)$ is unitary for a.e. $\mu \in \mathbb{R}$, then the spectrum of the selfadjoint operator $A=\operatorname{ker}\left(\Gamma_{0}\right)$ is purely singular and the absolutely continuous part $A^{a c}$ of $A$ is trivial.

Proof. (i) Observe that condition (5.1) together with $\|W(\eta)\| \leq 1$ implies that $\operatorname{ker}(I-W(\eta))=\{0\}$ holds for all $\eta \in \mathbb{C}_{+}$. Indeed, for $x \in \operatorname{ker}(I-W(\eta))$ we have $\|W(\eta) x\|=\|x\|$ and hence

$$
\left(\left(I-W(\eta)^{*} W(\eta)\right) x, x\right)=0
$$

which yields $x=0$. We define a function $M: \mathbb{C}_{+} \rightarrow\left[\mathbb{C}^{n}\right]$ by

$$
\mathbb{C}_{+} \ni \eta \mapsto M(\eta):=i(I+W(\eta))(I-W(\eta))^{-1}
$$

and we extend $M$ to the lower half-plane by $\mathbb{C}_{-} \ni \eta \mapsto M(\eta):=M(\bar{\eta})^{*}$. Then $M$ is analytic and a straightforward computation shows

$$
\Im \mathrm{m}(M(\eta))=\left(I-W(\eta)^{*}\right)^{-1}\left(I-W(\eta)^{*} W(\eta)\right)(I-W(\eta))^{-1} \geq 0 .
$$

for $\eta \in \mathbb{C}_{+}$. Hence $M$ is a Nevanlinna function and condition (5.1) implies $\operatorname{ker}(\Im m(M(\eta)))=\{0\}$. From condition (5.2) we obtain

$$
\text { s- } \lim _{y \rightarrow+\infty} \frac{1}{y} M(i y)=\text { s- } \lim _{y \rightarrow+\infty} \frac{i}{y}(I+W(i y))(I-W(i y))^{-1}=0 .
$$

By Theorem 2.3 there exists a separable Hilbert space $\mathfrak{H}$, a (in general nondensely defined) simple symmetric operator $S$ with deficiency indices $(n, n)$ and a boundary triplet $\Pi=\left\{\mathbb{C}^{n}, \Gamma_{0}, \Gamma_{1}\right\}$ for $S^{*}$ such that $M$ is the corresponding Weyl function. For $\eta \in \mathbb{C}_{+}$we have

$$
W(\eta)=I-2 i(M(\eta)+i)^{-1}, \quad \eta \in \mathbb{C}_{+} .
$$

Setting $D:=-i I$ we have $\sqrt{-\Im m(D)}=I$ and hence the representation (5.3) follows from (5.7).

(ii) From Corollary 4.6 one immediately gets that $W$ can be regarded as the LaxPhillips scattering matrix of the Lax-Phillips scattering system $\left\{L, \mathcal{D}_{-}, \mathcal{D}_{+}\right\}$.

(iii) Making use of (5.6) one easily verifies that the condition (5.4) yields

$$
\lim _{y \rightarrow \infty} y \Im \mathrm{m}(M(i y) h, h)=\infty, \quad h \in \mathcal{H} \backslash\{0\} .
$$

Hence the operator $S$ is densely defined by Theorem 2.3 .

(iv) We consider the analytic function $w(\eta):=\operatorname{det}(I-W(\eta)), \eta \in \mathbb{C}_{+}$. Since the limit $W(\lambda+i 0):=\lim _{y \rightarrow+0} W(\lambda+i y)$ exists for a.e. $\lambda \in \mathbb{R}$ the limit $w(\lambda+i 0):=\lim _{y \rightarrow+0} w(\lambda+i y)$ exist for a.e. $\lambda \in \mathbb{R}$, too. If the Lebesgue measure of the set $\{\lambda \in \mathbb{R}: w(\lambda+i 0)=0\}$ is different from zero, then $w(\eta) \equiv 0$ for all 
$\eta \in \mathbb{C}_{+}$by the Lusin-Privalov theorem [20, Section III] but this is impossible by assumption (5.1), cf. proof of (i). Hence, the set $\{\lambda \in \mathbb{R}: w(\lambda+i 0)=0\}$ has Lebesgue measure zero. Therefore, the operator $(I-W(\lambda+i 0))^{-1}$ exist for a.e. $\lambda \in \mathbb{R}$. Using (5.6) we find that $\lim _{y \rightarrow+0} \Im \mathrm{m}(M(\lambda+i y))=0$ for a.e. $\lambda \in \mathbb{R}$. By [11, Theorem 4.3(iii)] we get that the selfadjoint operator $A=\operatorname{ker}\left(\Gamma_{0}\right)$ has no absolutely continuous spectrum, i.e., the absolutely continuous part of $A$ is trivial.

We remark, that the representation (5.3) can also be obtained from [15, Proposition 7.5]. In fact, in the special case considered here some parts of the proof of Theorem 5.1 (i) coincide with the proof of [15, Proposition 7.5].

The Lax-Phillips scattering system and the selfadjoint dilation $L$ in Theorem 5.1 can be made more explicit. Let $W: \mathbb{C}_{+} \rightarrow\left[\mathbb{C}^{n}\right]$ be as in the assumptions of Theorem 5.1 and define the function $M$ by

$$
M(\eta)=i(I+W(\eta))(I-W(\eta))^{-1}, \quad \eta \in \mathbb{C}_{+}
$$

and $M(\eta)=M(\bar{\eta})^{*}$ as in (5.5). Then $M$ is $\left[\mathbb{C}^{n}\right]$-valued Nevanlinna function and hence $M$ admits an integral representation of the form

$$
M(\eta)=\alpha+\int_{\mathbb{R}}\left(\frac{1}{t-\eta}-\frac{t}{1+t^{2}}\right) d \Sigma(t), \quad \eta \in \mathbb{C} \backslash \mathbb{R},
$$

where $\alpha$ is a symmetric matrix and $t \mapsto \Sigma(t)$ is a $\left[\mathbb{C}^{n}\right]$-valued nondecreasing symmetric matrix function on $\mathbb{R}$ such that $\int\left(1+t^{2}\right)^{-1} d \Sigma(t) \in\left[\mathbb{C}^{n}\right]$. We note that due to condition (ii) in Theorem 5.1 the linear term in the integral representation (5.8) is absent. Let $L_{\Sigma}^{2}\left(\mathbb{R}, \mathbb{C}^{n}\right)$ be the Hilbert space of $\mathbb{C}^{n}$-valued functions as in [10, 18, 24]. It was shown in [24] that the mapping

$$
f \mapsto \int_{\mathbb{R}} d \Sigma(t) f(t)
$$

defined originally on the space $C_{0}\left(\mathbb{R}, \mathbb{C}^{n}\right)$ of (strongly) continuous functions with compact support admits a continuous extension to an operator from $L_{\Sigma}^{2}\left(\mathbb{R}, \mathbb{C}^{n}\right)$ into $\mathbb{C}^{n}$. According to [15, 24, the adjoint of the (in general nondensely defined) closed symmetric operator

$$
\begin{aligned}
(S f)(t) & =t f(t), \\
\operatorname{dom}(S) & =\left\{f \in L_{\Sigma}^{2}\left(\mathbb{R}, \mathbb{C}^{n}\right): t f(t) \in L_{\Sigma}^{2}\left(\mathbb{R}, \mathbb{C}^{n}\right), \int_{\mathbb{R}} d \Sigma(t) f(t)=0\right\},
\end{aligned}
$$

is given by the linear relation

$$
S^{*}=\left\{\left\{f(t)+\frac{t}{1+t^{2}} h, t f(t)-\frac{1}{1+t^{2}} h\right\}: f(t), t f(t) \in L_{\Sigma}^{2}\left(\mathbb{R}, \mathbb{C}^{n}\right), h \in \mathbb{C}^{n}\right\},
$$

and that $\left\{\mathbb{C}^{n}, \Gamma_{0}, \Gamma_{1}\right\}$, where

$$
\Gamma_{0} \hat{f}:=h \quad \text { and } \quad \Gamma_{1} \hat{f}:=\alpha h+\int_{\mathbb{R}} d \Sigma(t) f(t),
$$

$\hat{f}=\left\{f(t)+t\left(1+t^{2}\right)^{-1} h, t f(t)-\left(1+t^{2}\right)^{-1} h\right\} \in S^{*}$, is a boundary triplet for $S^{*}$ with corresponding Weyl function $M(\cdot)$. Note that here $A_{0}=\operatorname{ker}\left(\Gamma_{0}\right)$ is the usual maximal multiplication operator in $L_{\Sigma}^{2}\left(\mathbb{R}, \mathbb{C}^{n}\right)$. 
Corollary 5.2 Let $W: \mathbb{C}_{+} \rightarrow\left[\mathbb{C}^{n}\right]$ be a contractive analytic function which satisfies the conditions (5.1) and (5.2) in Theorem 5.1. Then there exists a symmetric matrix $\alpha \in\left[\mathbb{C}^{n}\right]$ and a $\left[\mathbb{C}^{n}\right]$-valued nondecreasing symmetric matrix function $\Sigma(\cdot)$ on $\mathbb{R}$ such that

$$
W(\mu)=I-2 i\left(\alpha+i+\int_{\mathbb{R}}\left(\frac{1}{t-\mu}-\frac{t}{1+t^{2}}\right) d \Sigma(t)\right)^{-1}
$$

holds for all $\mu \in \mathbb{C}_{+}$and $\int\left(1+t^{2}\right)^{-1} d \Sigma(t) \in\left[\mathbb{C}^{n}\right]$. The function $W(\cdot)$ coincides with the Lax-Phillips scattering matrix of the system $\left\{L, \mathcal{D}_{-}, \mathcal{D}_{+}\right\}$, where $\mathcal{D}_{ \pm}=$ $L^{2}\left(\mathbb{R}_{ \pm}, \mathbb{C}^{n}\right)$ and

$$
\begin{gathered}
L\left(\begin{array}{c}
\left.f(t)+\frac{t}{1+t^{2}} h\right)=\left(\begin{array}{c}
t f(t)-\frac{1}{1+t^{2}} h \\
i g^{\prime}
\end{array}\right), \begin{array}{c}
f(t), t f(t) \in L_{\Sigma}^{2}\left(\mathbb{R}, \mathbb{C}^{n}\right), h \in \mathbb{C}^{n}, \\
g \in W_{2}^{1}\left(\mathbb{R}_{-}, \mathbb{C}^{n}\right) \oplus W_{2}^{1}\left(\mathbb{R}_{+}, \mathbb{C}^{n}\right),
\end{array} \\
\operatorname{dom}(L)=\left\{\left(\begin{array}{c}
f(t)+\frac{t}{1+t^{2}} h \\
g
\end{array}\right): \frac{i}{\sqrt{2}}(g(0+)+g(0-))=\alpha h+\int d \Sigma(t) f(t)\right.
\end{array}\right\}
\end{gathered}
$$

is the minimal selfadjoint dilation in $L_{\Sigma}^{2}\left(\mathbb{R}, \mathbb{C}^{n}\right) \oplus L^{2}\left(\mathbb{R}, \mathbb{C}^{n}\right)$ of the maximal dissipative multiplication operator $B=\operatorname{ker}\left(\Gamma_{1}+i \Gamma_{0}\right)$ in $L_{\Sigma}^{2}\left(\mathbb{R}, \mathbb{C}^{n}\right)$.

\section{A Spectral representations and scattering ma- trix}

Let $A$ be a selfadjoint operator in the separable Hilbert space $\mathfrak{H}$ and let $E(\cdot)$ be the corresponding spectral measure defined on the $\sigma$-algebra $\mathcal{B}(\mathbb{R})$ of Borel subsets of $\mathbb{R}$. The absolutely continuous and singular part of the measure $E(\cdot)$ is denoted by $E^{a c}(\cdot)$ and $E^{s}(\cdot)$, respectively. If $C \in[\mathcal{H}, \mathfrak{H}]$ is a Hilbert-Schmidt operator, then by [6, Lemma I.11]

$$
\Sigma(\delta):=C^{*} E(\delta) C, \quad \delta \in \mathcal{B}(\mathbb{R}),
$$

is a trace class valued measure on $\mathcal{B}(\mathbb{R})$ of finite variation. This measure admits a unique decomposition

$$
\Sigma(\cdot)=\Sigma^{s}(\cdot)+\Sigma^{a c}(\cdot)
$$

into a singular measure $\Sigma^{s}(\cdot)=C^{*} E^{s}(\cdot) C$ and an absolutely continuous measure $\Sigma^{a c}(\cdot)=C^{*} E^{a c}(\cdot) C$. According to [6, Proposition I.13] the trace class valued function $\lambda \mapsto \Sigma(\lambda):=C^{*} E((-\infty, \lambda)) C$ admits a derivative $K(\lambda):=\frac{d}{d \lambda} \Sigma(\lambda) \geq 0$ in the trace class norm for a.e. $\lambda \in \mathbb{R}$ with respect to the Lebesgue measure $d \lambda$ and

$$
\Sigma^{a c}(\delta)=\int_{\delta} K(\lambda) d \lambda, \quad \delta \in \mathcal{B}(\mathbb{R})
$$

holds. By $\mathcal{H}_{\lambda}:=\overline{\operatorname{ran}(K(\lambda))} \subseteq \mathcal{H}$ we define a measurable family of subspaces in $\mathcal{H}$. Let $P(\lambda)$ be the orthogonal projection from $\mathcal{H}$ onto $\mathcal{H}_{\lambda}$ and define a measurable family of projections by

$$
(P f)(\lambda):=P(\lambda) f(\lambda), \quad f \in L^{2}(\mathbb{R}, d \lambda, \mathfrak{H}) .
$$

Then $P$ is an orthogonal projection in $L^{2}(\mathbb{R}, d \lambda, \mathcal{H})$ and we denote the range of $P$ by $L^{2}\left(\mathbb{R}, d \lambda, \mathcal{H}_{\lambda}\right)$. In the following we regard $L^{2}\left(\mathbb{R}, d \lambda, \mathcal{H}_{\lambda}\right)$ as the direct integral of the measurable family of subspaces $\left\{\mathcal{H}_{\lambda}\right\}_{\lambda \in \mathbb{R}}$. 
Lemma A.1 Let A, E, $C$ and $K(\lambda)$ be as above and assume that the absolutely continuous subspace $\mathfrak{H}^{a c}(A)$ satisfies the condition

$$
\mathfrak{H}^{a c}(A)=\operatorname{clospan}\left\{E^{a c}(\delta) \operatorname{ran}(C): \delta \in \mathcal{B}(\mathbb{R})\right\} .
$$

Then the linear extension of the mapping

$$
E^{a c}(\delta) C f \mapsto \chi_{\delta}(\lambda) \sqrt{K(\lambda)} f \text { for a.e. } \lambda \in \mathbb{R}, \quad f \in \mathcal{H},
$$

onto the dense subspace $\operatorname{span}\left\{E^{a c}(\delta) \operatorname{ran}(C): \delta \in \mathcal{B}(\mathbb{R})\right\}$ of $\mathfrak{H}^{a c}(A)$ admits a unique continuation to an isometric isomorphism from $\Phi: \mathfrak{H}^{a c}(E) \rightarrow$ $L^{2}\left(\mathbb{R}, d \lambda, \mathcal{H}_{\lambda}\right)$ such that

$$
\left(\Phi E^{a c}(\delta) g\right)(\lambda)=\chi_{\delta}(\lambda)(\Phi g)(\lambda), \quad g \in \mathfrak{H}^{a c}(A),
$$

holds for any $\delta \in \mathcal{B}(\mathbb{R})$.

Proof. For $f \in \mathcal{H}$ and $\delta \in \mathcal{B}(\mathbb{R})$ we have

$$
\left\|\chi_{\delta}(\cdot) \sqrt{K(\cdot)} f\right\|^{2}=\int_{\delta}\|\sqrt{K(\lambda)} f\|_{\mathcal{H}}^{2} d \lambda=\left\|E^{a c}(\delta) C f\right\|_{\mathfrak{H}}^{2}
$$

and hence the extension of the mapping A.1 onto the subspace $\operatorname{span}\left\{E^{a c}(\delta) \operatorname{ran}(C): \delta \in \mathcal{B}(\mathbb{R})\right\}$ is an isometry into $L^{2}\left(\mathbb{R}, d \lambda, \mathcal{H}_{\lambda}\right)$. Then the unique extension $\Phi: \mathfrak{H}^{a c}(A) \rightarrow L^{2}\left(\mathbb{R}, d \lambda, \mathcal{H}_{\lambda}\right)$ is isometric and it remains to show that $\Phi$ is onto. Suppose that there exists $h \in L^{2}\left(\mathbb{R}, d \lambda, \mathcal{H}_{\lambda}\right)$ such that

$$
0=\left(\Phi E^{a c}(\delta) C f, h\right)=\int_{\delta}(\sqrt{K(\lambda)} f, h(\lambda))_{\mathcal{H}} d \lambda
$$

holds for all $\delta \in \mathcal{B}(\mathbb{R})$ and $f \in \mathcal{H}$. This implies $(\sqrt{K(\lambda)} f, h(\lambda))_{\mathfrak{H}}=0$ for a.e. $\lambda \in \mathbb{R}$ and hence $h(\lambda) \perp \mathcal{H}_{\lambda}$ for a.e. $\lambda \in \mathbb{R}$, thus $h(\lambda)=0$ for a.e. $\lambda \in \mathbb{R}$. Hence $\Phi$ is surjective. The relation (A.2) for $\Phi$ follows from A.1.

From (A.2) we immediately get that the maximal multiplication operator $\mathcal{Q}$ in $L^{2}\left(\mathbb{R}, d \lambda, \mathcal{H}_{\lambda}\right)$,

$$
\begin{aligned}
(\mathcal{Q} f)(\lambda) & :=\lambda f(\lambda) \\
f \in \operatorname{dom}(\mathcal{Q}) & :=\left\{f \in L^{2}\left(\mathbb{R}, d \lambda, \mathcal{H}_{\lambda}\right): \lambda f(\lambda) \in L^{2}\left(\mathbb{R}, d \lambda, \mathcal{H}_{\lambda}\right)\right\} .
\end{aligned}
$$

satisfies $\mathcal{Q} \Phi=\Phi A^{a c}$ and $\varphi(\mathcal{Q}) \Phi=\Phi \varphi\left(A^{a c}\right)$ for any bounded Borel measurable function $\varphi(\cdot): \mathbb{R} \longrightarrow \mathbb{R}$. In other words, the direct integral $L^{2}\left(\mathbb{R}, d \lambda, \mathcal{H}_{\lambda}\right)$ performs a spectral representation of the absolutely continuous part $A^{a c}$ of the selfadjoint operator $A$.

Suppose now that $B$ is also a selfadjoint operator in $\mathfrak{H}$ and assume that the difference of the resolvents

$$
(B-i)^{-1}-(A-i)^{-1}
$$

is a trace class operator. Then the wave operators

$$
W_{ \pm}(B, A):=s-\lim _{t \rightarrow \pm \infty} e^{i t B} e^{-i t A} P^{a c}(A)
$$


exists and are complete; cf., e.g., 6, Theorem I.1]. The scattering operator $S_{A B}:=W_{+}(B, A)^{*} W_{-}(B, A)$ regarded as an operator in $\mathfrak{H}^{a c}(A)$ is unitary and commutes with $A$. Therefore there is a measurable family $\left\{S_{A B}(\lambda)\right\}_{\lambda \in \mathbb{R}}$ of unitary operators $S_{A B}(\lambda) \in\left[\mathcal{H}_{\lambda}\right]$ such that $S_{A B}$ is unitarily equivalent to the multiplication operator $\mathcal{S}_{A B}$ induced by $\left\{S_{A B}(\lambda)\right\}_{\lambda \in \mathbb{R}}$ in $L^{2}\left(\mathbb{R}, d \lambda, \mathcal{H}_{\lambda}\right)$, that is, $\mathcal{S}_{A B}=\Phi S_{A B} \Phi^{-1}$. The measurable family $\left\{S_{A B}(\lambda)\right\}_{\lambda \in \mathbb{R}}$ is called the scattering matrix of the scattering system $\{A, B\}$.

The following theorem on the representation of the scattering matrix is an important ingredient in the proof of Theorem 3.1] A detailed proof of Theorem A.2 will appear in a forthcoming paper.

Theorem A.2 Let $A$ and $B$ be selfadjoint operators in the separable Hilbert space $\mathfrak{H}$ and suppose that the resolvent difference admits the factorization

$$
(B-i)^{-1}-(A-i)^{-1}=\phi(A) C G C^{*}=Q C^{*},
$$

where $C \in[\mathcal{H}, \mathfrak{H}]$ is a Hilbert-Schmidt operator, $G \in[\mathcal{H}], \phi(\cdot): \mathbb{R} \rightarrow \mathbb{R}$ is a bounded continuous function and $Q=\phi(A) C G$. Assume that the condition

$$
\mathfrak{H}^{a c}(A)=\operatorname{clospan}\left\{E^{a c}(\delta) \operatorname{ran}(C): \delta \in \mathcal{B}(\mathbb{R})\right\}
$$

is satisfied and let $K(\lambda)=\frac{d}{d \lambda} C^{*} E((-\infty, \lambda)) C$ and $\mathcal{H}_{\lambda}=\overline{\operatorname{ran}(K(\lambda))}$ for a.e. $\lambda \in \mathbb{R}$.

Then $L^{2}\left(\mathbb{R}, d \lambda, \mathcal{H}_{\lambda}\right)$ is a spectral representation of $A^{a c}$ and the scattering matrix $\left\{S_{A B}(\lambda)\right\}_{\lambda \in \mathbb{R}}$ of the scattering system $\{A, B\}$ has the representation

$$
S_{A B}(\lambda)=I_{\mathcal{H}_{\lambda}}+2 \pi i\left(1+\lambda^{2}\right)^{2} \sqrt{K(\lambda)} Z(\lambda) \sqrt{K(\lambda)} \in\left[\mathcal{H}_{\lambda}\right]
$$

for a.e. $\lambda \in \mathbb{R}$, where

$$
Z(\lambda)=\frac{1}{\lambda+i} Q^{*} Q+\frac{\phi(\lambda)}{(\lambda+i)^{2}} G+Q_{B}(\lambda+i 0)
$$

and the limit $Q_{B}(\lambda+i 0):=\lim _{\varepsilon \rightarrow+0} Q^{*}(B-\lambda-i \varepsilon)^{-1} Q$ is taken in the HilbertSchmidt norm.

\section{Acknowledgment}

The second author thanks the Weierstrass Institute of Applied Analysis and Stochastics in Berlin for financial support and hospitality.

\section{References}

[1] V.M. Adamjan and D.Z. Arov, On a class of scattering operators and characteristic operator-functions of contractions, Dokl. Akad. Nauk SSSR 160 (1965), 9-12.

[2] V.M. Adamjan and D.Z. Arov, On scattering operators and contraction semigroups in Hilbert space, Dokl. Akad. Nauk SSSR 165 (1965), 9-12.

[3] V.M. Adamjan and D.Z. Arov, Unitary couplings of semi-unitary operators, Akad. Nauk Armjan. SSR Dokl. 43 (1966) No. 5, 257-263. 
[4] V.M. Adamjan and D.Z. Arov, Unitary couplings of semi-unitary operators, Mat. Issled. 1 (1966) vyp. 2, 3-64.

[5] V.M. Adamyan and B.S. Pavlov, Null-range potentials and M.G. Krein's formula for generalized resolvents, Zap. Nauchn. Semin. Leningr. Otd. Mat. Inst. Steklova 149 (1986) 7-23 (russian); translation in J. Sov. Math. 42 no.2 (1988) 1537-1550.

[6] H. Baumgärtel and M. Wollenberg, Mathematical Scattering Theory, Akademie-Verlag, Berlin, 1983.

[7] J. Behrndt, M.M. Malamud, and H. Neidhardt, Scattering theory for open quantum systems with finite rank coupling, Math. Phys. Anal. Geom. 10 (2007), 313-358.

[8] J. Behrndt, M.M. Malamud, and H. Neidhardt, Scattering matrices and Weyl function, Proc. London Math. Soc. 97 (2008), 568-598.

[9] J. Behrndt, M.M. Malamud, and H. Neidhardt, Trace formulae for dissipative and coupled scattering systems, Oper. Theory Adv. Appl. 188 (2008), $49-85$.

[10] Ju.M. Berezans'kiı̌, Expansions in Eigenfunctions of Selfadjoint Operators, AMS Translations of Mathematical Monographs Vol. 17, Providence, R.I., 1968.

[11] J.F. Brasche, M.M. Malamud, and H. Neidhardt, Weyl function and spectral properties of self-adjoint extensions, Integral Equations Oper. Theory 43 (2002), 264-289.

[12] J. Brüning, V. Geyler, and K. Pankrashkin, Spectra of self-adjoint extensions and applications to solvable Schrödinger operators, Rev. Math. Phys. 20 (2008), 1-70.

[13] V.A. Derkach and M.M. Malamud, On the Weyl function and Hermitian operators with gaps, Sov. Math. Dokl. 35 (1987), 393-398.

[14] V.A. Derkach and M.M. Malamud, Generalized resolvents and the boundary value problems for Hermitian operators with gaps, J. Funct. Anal. 95 (1991), $1-95$.

[15] V.A. Derkach and M.M. Malamud, The extension theory of Hermitian operators and the moment problem, J. Math. Sci. 73 (1995), 141-242.

[16] W.F. Donoghue, Monotone Matrix Functions and Analytic Continuation, Springer, Berlin-New York, 1974.

[17] J.B. Garnett, Bounded Analytic Functions, Academic Press, New YorkLondon, 1981.

[18] V.I. Gorbachuk and M.L. Gorbachuk, Boundary Value Problems for Operator Differential Equations, Mathematics and its Applications (Soviet Series) 48, Kluwer Academic Publishers Group, Dordrecht, 1991. 
[19] T. Kato, Perturbation Theory for Linear Operators, Grundlehren der Mathematischen Wissenschaften, Band 132, Springer, Berlin-New York, 1976.

[20] P. Koosis, Introduction to $H_{p}$ spaces. LMS Lecture Note Series Vol. 40, Cambridge University Press, Cambridge, 1980.

[21] H. Langer and B. Textorius, On generalized resolvents and Q-functions of symmetric linear relations (subspaces) in Hilbert space, Pacific J. Math. 72 (1977), 135-165.

[22] P.D. Lax and R.S. Phillips, Scattering Theory, Academic Press, New York, 1967.

[23] M.M. Malamud, On a formula for the generalized resolvents of a nondensely defined Hermitian operator, Ukraïn. Mat. Zh. 44 (1992), 1658-1688.

[24] M.M. Malamud and S. M. Malamud, Spectral theory of operator measures in a Hilbert space, St. Petersburg Math. J. 15 (2003), 1-53.

[25] B.S. Pavlov, Dilation theory and spectral analysis of nonselfadjoint differential operators, In Mathematical programming and related questions, Theory of operators in linear spaces (Russian), pages 3-69, Central. Èkonom. Mat. Inst. Akad. Nauk SSSR, Moscow, 1976.

[26] B.S. Pavlov, Spectral analysis of a dissipative singular Schrödinger operator in terms of a functional model, In Partial differential equations, VIII, volume 65 of Encyclopaedia Math. Sci., pages 87-153, Springer, Berlin, 1996.

[27] B. Sz.-Nagy and C. Foias, Harmonic Analysis of Operators on Hilbert Space, North-Holland Publishing Co., Amsterdam, 1970.

[28] J. Weidmann, Lineare Operatoren in Hilberträumen. Teil II: Anwendungen, B.G. Teubner, Stuttgart, 2003.

[29] D.R. Yafaev, Mathematical Scattering Theory: General Theory, AMS Translations of Mathematical Monographs Vol. 105, Providence, RI, 1992. 